\title{
The first law and Wald entropy formula of heterotic stringy black holes at first order in $\alpha^{\prime}$
}

\author{
Zachary Elgood, Tomás Ortín and David Pereñíguez \\ Instituto de Física Teórica UAM/CSIC, \\ C/ Nicolás Cabrera, 13-15, C.U. Cantoblanco, E-28049 Madrid, Spain \\ E-mail: zachary.elgood@uam.es, tomas.ortin@csic.es, \\ david.perenniguez@uam.es
}

ABSTRACT: We derive the first law of black hole mechanics in the context of the Heterotic Superstring effective action to first order in $\alpha^{\prime}$ using Wald's formalism. We carefully take into account all the symmetries of the theory and, as a result, we obtain a manifestly gaugeand Lorentz-invariant entropy formula in which all the terms can be computed explicitly. An entropy formula with these properties allows unambiguous calculations of macroscopic black-hole entropies to first order in $\alpha^{\prime}$ that can be reliably used in a comparison with the microscopic ones. Such a formula was still lacking in the literature.

In the proof we use momentum maps to define covariant variations and Lie derivatives and restricted generalized zeroth laws which state the closedness of certain differential forms on the bifurcation sphere and imply the constancy of the associated potentials on it.

We study the relation between our entropy formula and other formulae that have been used in the literature.

Keywords: Black Holes in String Theory, Classical Theories of Gravity, Differential and Algebraic Geometry

ArXiv EPrint: 2012.14892 


\section{Contents}

1 Introduction 1

2 The HST effective action at first order in $\alpha^{\prime} \quad 4$

3 Variations of the fields $\quad 7$

$\begin{array}{ll}3.1 \text { Gauge transformations } & 7\end{array}$

3.2 Gauge charges 8

3.2.1 Kalb-Ramond charge $\quad 8$

$\begin{array}{llr}3.2 .2 & \text { Yang-Mills charge } & 9\end{array}$

$\begin{array}{lll}3.2 .3 & \text { Lorentz charge } & 12\end{array}$

$\begin{array}{lll}3.3 & \text { The transformations under diffeomorphisms } & 13\end{array}$

$\begin{array}{lll}\text { 3.3.1 Lie-Lorentz derivatives } & 13\end{array}$

$\begin{array}{lll}3.3 .2 & \text { Lie-Yang-Mills derivatives } & 14\end{array}$

$\begin{array}{ll}\text { 3.3.3 The Kalb-Ramond field } & 15\end{array}$

$\begin{array}{ll}3.4 & \text { The Wald-Noether charge } \\ \end{array}$

4 Restricted generalized zeroth laws $\quad 18$

5 The first law 20

6 Wald entropy 22

$\begin{array}{lll}7 & \text { Discussion } & 23\end{array}$

\section{Introduction}

The interpretation of the black-hole entropy in terms of the degeneracy of string microstates is, beyond any doubt, one of the main achievements of String Theory [1]. This interpretation relies, on the one hand, on the correct identification of the black-hole charges in terms of branes whose presence affects the quantization of the string. On the other, it depends on a correct calculation of the macroscopic entropy. In simple cases, at leading order in $\alpha^{\prime}$, the identification of the field fluxes with the brane sources that would produce them is straightforward and, also, the macroscopic entropy is given by the Bekenstein-Hawking formula $S=\mathcal{A}_{\mathcal{H}} /\left(4 G_{N}\right)$, where $\mathcal{A}_{\mathcal{H}}$ is the area of the horizon. In more complicated cases, the couplings can make the identification of the brane sources through the charges more complicated [2] and, beyond leading order in $\alpha^{\prime}$, the presence of terms of higher order in the curvature and, in the Heterotic Superstring case, of complicated Yang-Mills (YM) and Lorentz Chern-Simons terms [3] can also make the calculation of the macroscopic entropy very difficult. This is the problem we will deal with in this paper. 
The standard method to calculate the black-hole entropy in theories of higher order in the curvature is to use Wald's formalism $[4,5]$, usually applying directly the Iyer-Wald prescription [6]. As we have recently discussed in refs. [7-9] (see also references therein), the Iyer-Wald prescription was derived assuming that all the fields of the theory behave as tensors under diffeomorphisms which, as matter of fact, is only true for the metric and uncharged scalars. All the fields of the Standard Model, except for the metric, have some kind of gauge freedom and do not transform as tensors under diffeomorphisms. Even the gravitational field, if it is described by a Vielbein instead of by a metric, has a gauge freedom, as it transforms under local Lorentz transformations. In theories with fermions, Vielbeins are necessary to work with the spinorial fields in curved space time.

This problem was first noticed and solved by Jacobson and Mohd in ref. [10] for the Einstein-Hilbert action written in terms of the Vielbein. The solution consists in going back to the basic formalism of $[4,5]$ and dealing carefully with the gauge (local Lorentz) symmetry. In practice, this means taking into account the gauge transformations induced by the diffeomorphisms on the Vielbein. This can be done, for instance, by defining a Lorentzcovariant Lie derivative (Lie-Lorentz derivative) which can be decomposed into a standard Lie derivative and a local Lorentz transformation and which, apart from being covariant under Local lorentz transformations, vanishes identically when the diffeomorphism is an isometry of the metric (see refs. $[11,12]^{1}$ which build on earlier work by Lichnerowicz, Kosmann and others [14-20]). The Lie-Lorentz derivative has recently been used to extend the proof of the first law of black mechanics to supergravity, including the spinorial fields, in ref. [21].

A more mathematically rigorous (and complicated) treatment based on the theory of principal bundles, that also applies to Yang-Mills fields, was given by Prabhu in ref. [22]. ${ }^{2}$ Apart from the mathematical complexity, this approach cannot be used to handle higherrank form fields such as the Kalb-Ramond (KR) field. For this reason, in ref. [8] we proposed a simpler alternative, based on the construction of covariant Lie derivatives of all the fields with gauge freedom (a Maxwell field in the case of ref. [8]). This construction is based on the introduction of momentum maps [12, 24] which play a crucial role in this paper and which we will define later. The Lie-Lorentz derivative can also be seen as based on the definition of a Lorentz momentum map. ${ }^{3}$

In ref. [27] we have shown how to use momentum maps to construct covariant Lie derivatives in the Heterotic Superstring Effective action compactified in a torus at zeroth order in $\alpha^{\prime}$. The KR field of that theory contains Abelian Chern-Simons terms ${ }^{4}$ which induce Nicolai-Townsend transformations of the 2-form [28]. These terms modify the definitions of the conserved charges which ultimately appear in the first law of black hole mechanics along the lines of the classical refs. [29-32].

In this paper we use the same technique quite extensively to deal with the variety of fields and couplings that occur in the Heterotic Superstring effective action at first order in $\alpha^{\prime}$ and prove the first law of black hole mechanics, identifying the entropy. As we are

\footnotetext{
${ }^{1}$ See also ref. [13] for a more mathematically rigorous point of view.

${ }^{2}$ See also ref. [23] for a different point of view on this problem.

${ }^{3}$ In refs. [25, 26], momentum maps emerge as "improved gauge transformations".

${ }^{4}$ Only the Kaluza-Klein and winding vector fields appear there at zeroth order in $\alpha^{\prime}$.
} 
going to see, the entropy formula obtained is manifestly gauge-invariant and contains only terms which are known and can be computed explicitly. This is the first entropy formula proposed for this theory that satisfies all these properties. It allows us to compute reliably the entropy of black hole solutions to first order in $\alpha^{\prime}$ and compare the result with the entropy computed through microstate counting. As we will show in the last section, it gives the same results as the non-gauge-invariant formulae used in refs. $[7,9,33]$ in certain basis. ${ }^{5}$ This confirms the values of the entropies obtained in those references, and shows why, in spite of the manifest deficiencies of the entropy formulae used, we obtained the correct result.

A very interesting aspect of the momentum maps is that they are related to the zeroth law of black hole mechanics and its generalizations. ${ }^{6}$ In the simplest case, the momentum map associated to a Maxwell field can be interpreted as the electrostatic potential. ${ }^{7}$ The generalized zeroth law states that it is constant over the black hole horizon [36]. The horizon's surface gravity, which is the subject of the zeroth law, is also related to the Lorentz momentum map. For higher-rank fields, Copsey and Horowitz [37] and, afterwards, Compère [38] proved a restricted form of the generalized zeroth law (restricted because it refers only to the bifurcation sphere) which follows from the closedness of certain differential forms on it. In ref. [27] we proved that these closed forms are related to the momentum maps and we will call these statements restricted generalized zeroth laws. Here we will extend the results of ref. [27] to YM and KR fields and to the more complicated couplings of the Heterotic Superstring effective action at first order in $\alpha^{\prime} .^{8}$

The restricted generalized zeroth laws play a crucial role in the proof of the first law and in the identification of the entropy and they are intimately related to the definitions of conserved charges. In Wald's formalism, the entropy is identified only after the terms $\sim \Phi \delta \mathcal{Q}$ have been identified in the first law. As in ref. [27], this identification requires the addition and subtraction of several terms as demanded by the definitions of the charges $\mathcal{Q}$ and the potentials $\Phi$ on account of the restricted generalized zeroth laws. However, in this case, some of the terms added and subtracted will be shown to contribute to the entropy.

This paper is organized as follows: in section 2 we introduce the effective action of the Heterotic Superstring to first order in $\alpha^{\prime}$ and find how it changes under an arbitrary variation of the fields, which allows us to determine the equations of motion. In section 3 we study how the fields change under gauge and general coordinate transformations. We construct variations of the fields that vanish when the parameters of the transformations generate a symmetry of the field configuration and we find the integrals that give the

\footnotetext{
${ }^{5}$ These results differ slightly from the results obtained in refs. [34, 35] using the Iyer-Wald prescription in the higher-dimensional action before dimensional reduction. As pointed out in ref. [2], the dependence on the Riemann tensor changes after dimensional reduction and the formulae in refs. [7, 9, 33] have been found using the dimensionally-reduced action. The formula that we give here does not suffer from any of these problems. See the discussion in section 7.

${ }^{6}$ This was first noticed by Prabhu, albeit in a completely different language [22].

${ }^{7}$ The Maxwell momentum map is defined in a gauge invariant form, and so is the electrostatic potential. This is in contrast wit the standard definitions of the electrostatic potential used in the literature.

${ }^{8}$ Some of these couplings have been discussed before in the literature, specially in ref. [39] (see also references therein). See the discussion in section 7 .
} 
associated conserved charges. The conserved charge associated to the invariance under diffeomorphisms is the Wald-Noether charge. As we have discussed, the correct identification of the conserved charges is essential to obtain for the correct identification of the entropy in the first law. In section 4 we discuss the restricted generalized zeroth laws of this theory, which also play an essential role in the proof of the first law. In section 5 we prove the first law using the results obtained in the previous sections, which leads us to identify the Wald entropy formula in section 6 . Section 7 contains a discussion of our results, comparing them with the existing literature.

\section{The HST effective action at first order in $\alpha^{\prime}$}

The Heterotic Superstring effective action can be described at first order in $\alpha^{\prime}$ as follows [3]: ${ }^{9}$ we start by defining the zeroth-order KR field strength $H^{(0)}$ and its components $H^{(0)}{ }_{\mu \nu \rho}$ as

$$
H^{(0)} \equiv d B=\frac{1}{3 !} H_{\mu \nu \rho} d x^{\mu} \wedge d x^{\mu} \wedge d x^{\rho},
$$

where $B=\frac{1}{2} B_{\mu \nu} d x^{\mu} \wedge d x^{\mu}$ is the KR 2-form potential. Then, if $\omega^{a b}=\omega_{\mu}^{a b} d x^{\mu}$ is the Levi-Civita spin connection, ${ }^{10}$ we define the zeroth-order torsionful spin connections ${ }^{11}$

$$
\Omega_{( \pm) a b}^{(0)}=\omega_{a b} \pm \frac{1}{2} \imath_{b} \imath_{a} H^{(0)}
$$

and their corresponding zeroth-order curvature 2-forms and Chern-Simons 3-forms

$$
\begin{aligned}
& R_{( \pm)}^{(0) a b} \equiv d \Omega_{( \pm)}^{(0) a b}-\Omega_{( \pm)}^{(0) a} \wedge \Omega_{( \pm)}^{(0) c b}, \\
& \omega_{( \pm)}^{(0)}=R_{( \pm)}^{(0) a} \wedge \Omega_{( \pm)}^{(0) b} a+\frac{1}{3} \Omega_{( \pm) b}^{(0) a} \wedge \Omega_{( \pm)}^{(0) b} \wedge \wedge \Omega_{( \pm)}^{(0) c} a .
\end{aligned}
$$

Next, we define the gauge field strength 2-form and the Chern-Simons 3-forms for the YM field $A^{A}=A_{\mu}^{A} d x^{\mu}$ by

$$
\begin{aligned}
F^{A} & =d A^{A}+\frac{1}{2} f_{B C}{ }^{A} A^{B} \wedge A^{C}, \\
\omega^{\mathrm{YM}} & =F_{A} \wedge A^{A}-\frac{1}{6} f_{A B C} A^{A} \wedge A^{B} \wedge A^{C},
\end{aligned}
$$

where we have lowered the adjoint group indices $A, B, C, \ldots$ in the structure constants $f_{A B}{ }^{C}$ and gauge fields using the Killing metric.

Then, we can define the first-order KR field strength 3-form as

$$
H^{(1)} \equiv H^{(0)}+\frac{\alpha^{\prime}}{4}\left(\omega^{\mathrm{YM}}+\omega_{(-)}^{(0)}\right) .
$$

\footnotetext{
${ }^{9}$ We use the conventions of ref. [12], reviewed for the zeroth-order case in ref. [27]. In particular, the relation with the fields in ref. [3] can be found in ref. [40].

${ }^{10}$ If $e^{a}=e^{a}{ }_{\mu} d x^{\mu}$ are the Vielbein, the spin connection is defined to satisfy the Cartan structure equation $\mathcal{D} e^{a} \equiv d e^{a}-\omega^{a}{ }_{b} \wedge e^{b}=0$.

${ }^{11}$ We denote by $\imath_{a} A$ the inner product of $e_{a} \equiv e_{a}{ }^{\mu} \partial_{\mu}\left(e_{a}{ }^{\mu} e^{b}{ }_{\mu}=\delta^{a}{ }_{b}\right)$ with the differential form $A$. If $A$ is a $p$-form with components $A_{\mu_{1} \cdots \mu_{p}}, \imath_{a} A$ is the $(p-1)$ form with components $e_{a}{ }^{\nu} A_{\nu \mu_{1} \cdots \mu_{p-1}}$.
} 
Its Bianchi identity takes the well-known form

$$
d H^{(1)}=\frac{\alpha^{\prime}}{4}\left(F_{A} \wedge F^{A}+R_{(-)}^{(0)} a_{b} \wedge R_{(-)}^{(0) b} a\right) .
$$

Having made these definitions and adding the dilaton field $\phi$, we can write the Heterotic Superstring effective action to first-order in $\alpha^{\prime}$ as

$$
\begin{aligned}
S^{(1)}\left[e^{a}, B, A^{A}, \phi\right]= & \frac{g_{s}^{(d) 2}}{16 \pi G_{N}^{(d)}} \int e^{-2 \phi}\left[(-1)^{d-1} \star\left(e^{a} \wedge e^{b}\right) \wedge R_{a b}-4 d \phi \wedge \star d \phi\right. \\
& \left.+\frac{1}{2} H^{(1)} \wedge \star H^{(1)}+(-1)^{d} \frac{\alpha^{\prime}}{4}\left(F_{A} \wedge \star F^{A}+R_{(-)}^{(0) a} b \wedge \star R_{(-)}^{(0)} b\right)\right] \\
\equiv & \int \mathbf{L}^{(1)} .
\end{aligned}
$$

Although this action is defined in 10 dimensions, we have left the dimension arbitrary $(d)$ because that allows us to use the results in other dimensions after trivial dimensional reduction on a torus. In this action, $G_{N}^{(d)}$ is the $d$-dimensional Newton constant and $g_{s}^{(d)}$ is the $d$-dimensional string coupling constant, identified with the vacuum expectation value of the exponential of the $d$-dimensional dilaton field $g_{s}^{(d)}=<e^{\phi}>$. In solutions such as black holes that asymptote to a vacuum solution at infinity $e^{\phi} \rightarrow e^{\phi_{\infty}}=<e^{\phi}>=g_{s}^{(d)}$.

This is a very complex action. Due to this complexity and to the lemma proven in ref. [3] which we will explain later, it is convenient to perform a general variation of the action in two steps: first, we only vary the action with respect to the explicit occurrences of the fields, where we define "explicit occurrences" as those which do not take place in the torsionful spin connection $\Omega_{(-)}^{(0)}$. Then, we vary the action with respect to the occurrences of the fields via $\Omega_{(-)}^{(0)}$ using the chain rule. All the occurrences of the dilaton and YM fields are explicit, but those of the Vielbein and KR field are not, because they (and only they) are present in $\Omega_{(-)}^{(0)}$.

Thus, setting $g_{s}^{(d) 2}\left(16 \pi G_{N}^{(d)}\right)^{-1}=1$ for the time being in order to simplify the formulae, we find that under a general variation of the "explicit" occurrences of the fields, the action transforms as follows:

$$
\begin{aligned}
\delta_{\exp } S^{(1)}=\int & \left\{\mathbf{E}_{\exp a}^{(1)} \wedge \delta e^{a}+\mathbf{E}_{\exp B}^{(1)} \wedge \delta B+\mathbf{E}_{\phi}^{(1)} \delta \phi+\mathbf{E}_{A}^{(1)} \delta A^{A}\right. \\
& \left.+d \mathbf{\Theta}_{\exp }^{(1)}(\varphi, \delta \varphi)\right\}
\end{aligned}
$$

where $\varphi$ stands for all the fields of the theory,

$$
\begin{aligned}
\mathbf{E}_{\exp a}^{(1)}= & e^{-2 \phi} \imath_{a} \star\left(e^{c} \wedge e^{d}\right) \wedge R_{c d}-2 \mathcal{D}\left(\imath_{b} d e^{-2 \phi}\right) \wedge \star\left(e^{b} \wedge e^{c}\right) g_{c a} \\
& +(-1)^{d-1} 4 e^{-2 \phi}\left(\imath_{a} d \phi \star d \phi+d \phi \wedge \imath_{a} \star d \phi\right) \\
& +\frac{(-1)^{d}}{2} e^{-2 \phi}\left(\imath_{a} H^{(1)} \wedge \star H^{(1)}+H^{(1)} \wedge \imath_{a} \star H^{(1)}\right) \\
& +\frac{\alpha^{\prime}}{4} e^{-2 \phi}\left(\imath_{a} F_{A} \wedge \star F^{A}-F_{A} \wedge \imath_{a} \star F^{A}\right. \\
& \left.+\imath_{a} R_{(-)}^{(0) b}{ }^{\prime} \wedge \star R_{(-)}^{(0) c} b-R_{(-)}^{(0) b} \wedge \imath_{a} \star R_{(-)}^{(0)} b\right)
\end{aligned}
$$




$$
\begin{aligned}
\mathbf{E}_{\exp B}^{(1)} & =-d\left(e^{-2 \phi} \star H^{(1)}\right) \\
\mathbf{E}_{\phi}^{(1)} & =8 d\left(e^{-2 \phi} \star d \phi\right)-2 \mathbf{L}^{(1)}, \\
\mathbf{E}_{A}^{(1)} & =-\frac{\alpha^{\prime}}{2}\left\{\mathcal{D}\left(e^{-2 \phi} \star F_{A}\right)+(-1)^{d} e^{-2 \phi} \star H^{(0)} \wedge F_{A}\right\}-\frac{\alpha^{\prime}}{4} \mathbf{E}_{\exp B}^{(1)} \wedge A_{A},
\end{aligned}
$$

and

$$
\begin{aligned}
\boldsymbol{\Theta}_{\exp }^{(1)}(\varphi, \delta \varphi)= & -e^{-2 \phi} \star\left(e^{a} \wedge e^{b}\right) \wedge \delta \omega_{a b}+2 \imath_{a} d e^{-2 \phi} \star\left(e^{a} \wedge e^{b}\right) \wedge \delta e_{b}-8 e^{-2 \phi} \star d \phi \delta \phi \\
& +e^{-2 \phi} \star H^{(1)} \wedge \delta B+\frac{\alpha^{\prime}}{2} e^{-2 \phi}\left(\star F_{A}-\frac{1}{2} \star H^{(1)} \wedge A_{A}\right) \wedge \delta A^{A} .
\end{aligned}
$$

An alternative form of the YM equations that arises in the calculations is

$$
\mathbf{E}_{A}^{(1)}=-\frac{\alpha^{\prime}}{2} \mathcal{D}\left(e^{-2 \phi} \star F_{A}-e^{-2 \phi} \star H^{(0)} \wedge A_{A}\right)+(-1)^{d-1} \frac{\alpha^{\prime}}{4} e^{-2 \phi} \star H^{(0)} \wedge d A_{A} .
$$

Observe that neither the YM equations of motion transform covariantly nor is $\boldsymbol{\Theta}_{\exp }^{(1)}$ invariant under YM gauge transformations. For the YM equations this is not a big problem since the troublesome term is proportional to the KR equation of motion, but there is no obvious fix for the pre-symplectic potential. Nevertheless, we will see that, in the end, we will get gauge-invariant charges and, in particular a gauge-invariant Wald-Noether charge.

An important property of the HST effective action is that the YM fields and the torsionful spin connection occur on exactly the same footing [41]. The variation of the action with respect to the torsionful spin connection takes exactly the same form as the YM equation, the only difference being the group indices and their contractions. Thus,

$$
\begin{aligned}
& \delta S^{(1)}=\int\left\{\mathbf{E}_{\exp a}^{(1)} \wedge \delta e^{a}+\mathbf{E}_{\exp B}^{(1)} \wedge \delta B+\mathbf{E}_{\phi}^{(1)} \delta \phi+\mathbf{E}_{A}^{(1)} \wedge \delta A^{A}+\mathbf{E}^{(1) b}{ }_{a} \wedge \delta \Omega_{(-)}^{(0) a} b\right. \\
&\left.+d \mathbf{\Theta}^{(1)}(\varphi, \delta \varphi)\right\},
\end{aligned}
$$

where the variation with respect to the torsionful spin connection is given by

$$
\mathbf{E}^{(1) b}{ }_{a}=-\frac{\alpha^{\prime}}{2}\left\{\mathcal{D}_{(-)}\left(e^{-2 \phi} \star R_{(-)}^{(0) b} a\right)+(-1)^{d} e^{-2 \phi} \star H^{(0)} \wedge R_{(-)}^{(0) b} a\right\}-\frac{\alpha^{\prime}}{4} \mathbf{E}_{\exp B}^{(1)} \wedge \Omega_{(-)}^{(0) b} a,
$$

or

$$
\mathbf{E}^{(1) b}{ }_{a}=-\frac{\alpha^{\prime}}{2} \mathcal{D}_{(-)}\left(e^{-2 \phi} \star R_{(-)}^{(0) b} a-e^{-2 \phi} \star H^{(0)} \wedge \Omega_{(-)}^{(0) b}\right)+(-1)^{d-1} \frac{\alpha^{\prime}}{4} \star H^{(0)} \wedge d \Omega_{(-)}^{(0) b},
$$

and the pre-symplectic $(d-1)$-form is given by

$$
\boldsymbol{\Theta}^{(1)}(\varphi, \delta \varphi)=\boldsymbol{\Theta}_{\exp }^{(1)}(\varphi, \delta \varphi)+\frac{\alpha^{\prime}}{2} e^{-2 \phi}\left(\star R_{(-)}^{(0) b} a-\frac{1}{2} \star H^{(1)} \wedge \Omega_{(-)}^{(0) b} a\right) \wedge \delta \Omega_{(-)}^{(0) a} b,
$$

with $\boldsymbol{\Theta}_{\exp }^{(1)}(\varphi, \delta \varphi)$ given in eq. (2.11).

The parallelism between the YM and torsionful spin connection terms also leads to the same problems of non-covariance of $\mathbf{E}^{(1) b}{ }_{a}$ and non-invariance of the additional term in $\boldsymbol{\Theta}^{(1)}$. 
An important difference between the equations of motion of these two connections is that, according to the lemma proven in ref. [3], $\mathbf{E}^{(1) a_{b}}$ is proportional to $\alpha^{\prime}$ and to a combination of the zeroth-order equations $\mathbf{E}_{a}^{(0)}, \mathbf{E}_{B}^{(0)}$ and $\mathbf{E}_{\phi}^{(0)}$. This means that field configurations that solve the equations $\mathbf{E}_{\exp a}^{(1)}=0, \mathbf{E}_{\exp B}^{(1)}=0, \mathbf{E}_{\phi}^{(1)}=0$ and $\mathbf{E}_{A}^{(1)}=0$ are solutions of the complete first-order equations, to that order in $\alpha^{\prime}$. This crucial property effectively reduces the degree of the differential equations to 2 , avoiding the problems that arise with dynamical equations that involve derivatives of the fields of higher order.

\section{Variations of the fields}

It is convenient to start by describing the gauge transformations of the fields and the associated Noether identities to be able to compute the associated conserved charges. Afterwards, we will discuss the transformations of the fields under diffeomorphisms and the associated Wald-Noether charge.

\subsection{Gauge transformations}

The fields occurring in the effective action eq. (2.8) transform under 3 kinds of gauge transformations:

1. KR gauge transformations with 1 -form parameter $\Lambda, \delta_{\Lambda}$, which only act on $B$.

2. YM gauge transformations with parameter $\chi^{A}, \delta_{\chi}$, which act on the YM fields and on $B$ as Nicolai-Townsend transformations.

3. Local Lorentz transformations with parameter $\sigma^{a b}, \delta_{\sigma}$, which act on the Vielbein and induce transformations of spin connections and curvature and which also act on $B$ as Nicolai-Townsend transformations.

The transformation rules are

$$
\begin{aligned}
& \delta_{\sigma} e^{a}=\sigma_{b}^{a} e^{b}, \\
& \delta_{\chi} A^{A}=\mathcal{D} \chi^{A} \equiv d \chi^{A}+f_{B C}{ }^{A} A^{B} \chi^{C}, \\
& \delta B=\left(\delta_{\Lambda}+\delta_{\chi}+\delta_{\sigma}\right) B=d \Lambda-\frac{\alpha^{\prime}}{4} \chi_{A} d A^{A}-\frac{\alpha^{\prime}}{4} \sigma_{b}^{a} d \Omega_{(-)}^{(0)} b .
\end{aligned}
$$

The induced local Lorentz transformations of the connections are

$$
\begin{aligned}
\delta_{\sigma} \omega^{a b} & =\mathcal{D} \sigma^{a b}=d \sigma^{a b}-2 \omega^{[a \mid}{ }_{c} \sigma^{c \mid b]}, \\
\delta_{\sigma} \Omega_{(-)}^{(0)} a b & =\mathcal{D}_{(-)}^{(0)} \sigma^{a b}=d \sigma^{a b}-2 \Omega_{(-)}^{(0)}{ }_{c}{ }_{c} \sigma^{c \mid b]},
\end{aligned}
$$

and the transformations of the curvatures are

$$
\begin{aligned}
\delta_{\chi} F^{A} & =-\chi^{B} f_{B C}{ }^{A} F^{C} \\
\delta_{\sigma} R^{a b} & =2 \sigma^{[a \mid}{ }_{c} R^{c \mid b]} . \\
\delta_{\sigma} R_{(-)}^{(0)} a b & =2 \sigma^{[a \mid}{ }_{c} R_{(-)}^{(0)}{ }^{c \mid b]} .
\end{aligned}
$$


Finally, for the sake of completeness and their later use, we quote the gauge transformations of the Chern-Simons 3-forms

$$
\begin{aligned}
& \delta_{\chi} \omega^{\mathrm{YM}}=\frac{\alpha^{\prime}}{4} d\left(\chi_{A} d A^{A}\right), \\
& \delta_{\sigma} \omega_{(-)}^{(0)}=+\frac{\alpha^{\prime}}{4} d\left(\sigma_{b}^{a} d \Omega_{(-)}^{(0)} b\right),
\end{aligned}
$$

and the Ricci identities

$$
\begin{gathered}
\mathcal{D} \mathcal{D} \chi^{A}=-f_{B C}{ }^{A} \chi^{B} F^{C}=\delta_{\chi} F^{A} \\
\mathcal{D}_{(-)}^{(0)} \mathcal{D}_{(-)}^{(0)} \sigma^{a b}=-2 R_{(-)}^{(0)}{ }^{[a \mid}{ }_{c} \sigma^{c \mid b]}=\delta_{\sigma} R_{(-)}^{(0)} a b
\end{gathered}
$$

The exact invariance of the action $S^{(1)}$ in eq. (2.8) under the above gauge transformations leads, in a rather trivial way, to the following Noether identities [40]

$$
\begin{aligned}
d \mathbf{E}_{\exp B}^{(1)} & =0, \\
\mathcal{D} \mathbf{E}_{A}^{(1)}+(-1)^{d-1} \frac{\alpha^{\prime}}{4} \mathbf{E}_{\exp B}^{(1)} \wedge d A_{A} & =0, \\
\mathcal{D}_{(-)}^{(0)} \mathbf{E}^{(1)} b^{a}+(-1)^{d-1} \frac{\alpha^{\prime}}{4} \mathbf{E}_{\exp B}^{(1)} \wedge d \Omega_{(-) b}^{(0)} a & =0, \\
\mathbf{E}_{\exp }^{(1)[a} \wedge e^{b]}+\frac{\alpha^{\prime}}{4} \mathbf{E}_{\exp B}^{(1)} \wedge d \Omega^{(0) a b}+(-1)^{d-1} \mathcal{D}_{(-)}^{(0)} \mathbf{E}^{(1) a b} & =0 .
\end{aligned}
$$

Eq. (3.6c) is just a particular case of eq. (3.6b) with adjoint Lorentz indices. Furthermore, the last two identities imply the symmetry of the Einstein equation, which in the language $\mathrm{f}$ differential forms and Vielbeins, is expressed in the form

$$
\mathbf{E}_{\exp }^{(1)[a} \wedge e^{b]}=0 .
$$

\subsection{Gauge charges}

For the sake of simplicity, we are going to start by the charge associated to the $\delta_{\Lambda}$ transformations, that we are going to call Kalb-Ramond charge.

\subsubsection{Kalb-Ramond charge}

Let us consider the transformation of the action eq. (2.8) under the gauge transformations $\delta_{\Lambda}$. Taking into account that this symmetry only acts on $B,{ }^{12}$ eqs. (2.13) and (2.16) we get

$$
\delta_{\Lambda} S^{(1)}=\int\left\{\mathbf{E}_{\exp B}^{(1)} \wedge d \Lambda+d\left[e^{-2 \phi} \star H^{(1)} \wedge d \Lambda\right]\right\} .
$$

Integrating by parts the first term and using the Noether identity eq. (3.6a)

$$
\delta_{\Lambda} S^{(1)}=\int d\left\{(-1)^{d} \mathbf{E}_{\exp B}^{(1)} \wedge \Lambda+e^{-2 \phi} \star H^{(1)} \wedge d \Lambda\right\} \equiv \int d \mathbf{J}[\Lambda]
$$

\footnotetext{
${ }^{12}$ We consider the variation of the torsionful spin connection to be zero under this transformation.
} 
Since $\delta_{\Lambda} S^{(1)}=0$, the integrand must vanish, which means that $\mathbf{J}[\Lambda]$ must be locally exact. Indeed,

$$
\mathbf{J}[\Lambda]=d \mathbf{Q}[\Lambda], \quad \text { with } \quad \mathbf{Q}[\Lambda]=\Lambda \wedge\left(e^{-2 \phi} \star H^{(1)}\right) .
$$

Integrating the $(d-2)$-form $\mathbf{Q}[\Lambda]$ over $(d-2)$-dimensional compact surfaces $\mathcal{S}_{d-2}$ for $\Lambda \mathrm{s}$ that leave the KR field $B$ invariant we get conserved charges associated to those $\Lambda$ s. These $\Lambda$ s are simply closed 1-forms. ${ }^{13}$ The Hodge decomposition theorem allows us to write each of them as the sum of an exact and a harmonic form that we denote by $\Lambda_{e}$ and $\Lambda_{h}$, respectively. On-shell, the exact form $\Lambda_{e}=d \lambda$ will not contribute to the integral and the charge will be given by

$$
\mathcal{Q}\left(\Lambda_{h}\right)=\int_{\mathcal{S}_{d-2}} \Lambda_{h} \wedge\left(e^{-2 \phi} \star H\right)
$$

Now we can use duality between homology and cohomology: if $C_{\Lambda_{h}}$ is the $(d-3)$-cycle dual to $\Lambda_{h}$ we arrive at the charges

$$
\mathcal{Q}\left(\Lambda_{h}\right)=-\frac{g_{s}^{(d) 2}}{16 \pi G_{N}^{(d)}} \int_{C_{\Lambda_{h}}} e^{-2 \phi} \star H,
$$

where we have recovered the factor of $g_{s}^{(d) 2}\left(16 \pi G_{N}^{(d)}\right)^{-1}$ and added a conventional sign.

\subsubsection{Yang-Mills charge}

Now, let us consider the charges associated to the YM gauge transformations $\delta_{\chi}$. Again, from eqs. (2.13) and (2.16), taking into account that this symmetry acts on the YM fields $A^{A}$ but also on the KR 2-form $B$, we have

$$
\begin{aligned}
\delta_{\chi} S^{(1)}= & \int\left\{\mathbf{E}_{\exp B}^{(1)} \wedge \delta_{\chi} B+\mathbf{E}_{A}^{(1)} \wedge \delta_{\chi} A^{A}\right. \\
& \left.+d\left[e^{-2 \phi} \star H^{(1)} \wedge \delta_{\chi} B+\frac{\alpha^{\prime}}{2} e^{-2 \phi}\left(\star F_{A}-\frac{1}{2} \star H^{(1)} \wedge A_{A}\right) \wedge \delta_{\chi} A^{A}\right]\right\} .
\end{aligned}
$$

The parameters $\chi^{A}$ that we will use are those that preserve the field configuration, leaving $A^{A}$ and $B$ invariant. The YM fields are left invariant by covariantly constant $\chi^{A} \mathrm{~s}$, i.e. $\chi^{A_{\mathrm{s}}}$ that we will denote by $\kappa^{A}$ satisfying

$$
\mathcal{D} \kappa^{A}=0
$$

We can call these parameters vertical Killing vector fields from he principal bundle point of view, with the standard Killing vectors of the base manifold playing the rôle of horizontal Killing vector fields.

\footnotetext{
${ }^{13}$ Here we follow refs. [37, 38]. This discussion is identical to the discussion we made for the zeroth-order case in ref. [27].
} 
The integrability condition of the vertical Killing vector equation is, according to eq. (3.5a),

$$
\delta_{\kappa} F^{A}=-f_{B C}{ }^{A} \kappa^{B} F^{C}=0,
$$

so they also leave the field strengths invariant, as expected.

The vertical Killing vector fields $\kappa^{A} \mathrm{~s}$ will not leave $B$ invariant, though, but we can rewrite the transformation in the form

$$
\delta_{\kappa} B=-\frac{\alpha^{\prime}}{4} \kappa_{A} d A^{A}=-\frac{\alpha^{\prime}}{2} \kappa_{A} F^{A}+d\left(\frac{\alpha^{\prime}}{4} \kappa_{A} A^{A}\right) .
$$

Now we observe that, due to the YM Bianchi identity $\mathcal{D} F^{A}=0, \kappa_{A} F^{A}$ is a closed 2-form and, locally, there is a 1 -form $\Psi_{\kappa}$ such that

$$
d \Psi_{\kappa}=-\kappa_{A} F^{A}
$$

and which we will call vertical YM momentum map. ${ }^{14}$

Then, we define the parameter of a compensating $\Lambda$ transformation

$$
\Lambda_{\chi}=-\frac{\alpha^{\prime}}{2} \Psi_{\chi}-\frac{\alpha^{\prime}}{4} \chi_{A} A^{A}
$$

where $\Psi_{\chi}$ is a 1-form such that, when $\chi^{A}=\kappa^{A}$ (i.e. when it is a vertical Killing vector field), it satisfies eq. (3.17). Combining the original $\delta_{\chi}$ transformation with the compensating $\delta_{\Lambda_{\chi}}$ transformation we find a new $\delta_{\chi} B$ that vanishes for covariantly constant $\chi^{A_{\mathrm{s}}}$ :

$$
\delta_{\chi} B \equiv-\frac{\alpha^{\prime}}{2}\left(d \Psi_{\chi}+\chi_{A} F^{A}\right)-\frac{\alpha^{\prime}}{4} \mathcal{D} \chi_{A} \wedge A^{A}
$$

The vanishing of $\delta_{\chi} B$ for covariantly constant $\chi^{A} \mathrm{~s}$ is gauge invariant because

$$
\delta_{\chi^{\prime}} \delta_{\chi} \sim \mathcal{D} \chi
$$

Substituting the transformation eq. (3.19) and the standard gauge transformation of the YM fields into eq. (3.13) we get

$$
\begin{aligned}
\delta_{\chi} S^{(1)}= & \int\left\{\mathbf{E}_{A}^{(1)} \wedge \mathcal{D} \chi^{A}+\mathbf{E}_{\exp B}^{(1)} \wedge\left[-d\left(\frac{\alpha^{\prime}}{2} \Psi_{\chi}+\frac{\alpha^{\prime}}{4} \chi_{A} A^{A}\right)-\frac{\alpha^{\prime}}{4} \chi_{A} d A^{A}\right]\right. \\
& +d\left\{e^{-2 \phi} \star H^{(1)} \wedge\left[-d\left(\frac{\alpha^{\prime}}{2} \Psi_{\chi}+\frac{\alpha^{\prime}}{4} \chi_{A} A^{A}\right)-\frac{\alpha^{\prime}}{4} \chi_{A} d A^{A}\right]\right. \\
& \left.\left.+\frac{\alpha^{\prime}}{2} e^{-2 \phi}\left(\star F_{A}-\frac{1}{2} \star H^{(1)} \wedge A_{A}\right) \wedge \mathcal{D} \chi^{A}\right\}\right\} .
\end{aligned}
$$

\footnotetext{
${ }^{14}$ Compare this equation with the equation satisfied by the standard (horizontal) YM momentum map eq. (3.43).
} 
Integrating by parts the first terms and combining the different terms in an appropriate way we can rewrite the variation in the form

$$
\begin{aligned}
\delta_{\chi} S^{(1)}= & \int\left\{(-1)^{d} \chi^{A}\left(\mathcal{D} \mathbf{E}_{A}^{(1)}+(-1)^{d-1} \frac{\alpha^{\prime}}{4} \mathbf{E}_{\exp B}^{(0)} \wedge d A_{A}\right)\right. \\
& -\left(\frac{\alpha^{\prime}}{2} \Psi_{\chi}+\frac{\alpha^{\prime}}{4} \chi_{A} A^{A}\right) \wedge d \mathbf{E}_{\exp B}^{(0)} \\
& +d\left\{(-1)^{d-1} \chi^{A}\left(\mathbf{E}_{A}^{(1)}+(-1)^{d} \frac{\alpha^{\prime}}{4} e^{-2 \phi} \star H^{(0)} \wedge d A_{A}\right)\right. \\
& -\left(\frac{\alpha^{\prime}}{2} \Psi_{\chi}+\frac{\alpha^{\prime}}{4} \chi_{A} A^{A}\right) \wedge \mathbf{E}_{\exp B}^{(0)} \\
& +e^{-2 \phi} \star H^{(1)} \wedge\left[-d\left(\frac{\alpha^{\prime}}{2} \Psi_{\chi}+\frac{\alpha^{\prime}}{4} \chi_{A} A^{A}\right)\right] \\
& \left.\left.+\frac{\alpha^{\prime}}{2} e^{-2 \phi}\left(\star F_{A}-\frac{1}{2} \star H^{(1)} \wedge A_{A}\right) \wedge \mathcal{D} \chi^{A}\right\}\right\}
\end{aligned}
$$

The terms in the first and second lines vanish identically because of the Noether identities eqs. (3.6b) and (3.6a), respectively, and we arrive at

$$
\begin{aligned}
\delta_{\chi} S^{(1)}= & \int d\left\{(-1)^{d-1} \chi^{A}\left(\mathbf{E}_{A}^{(1)}+(-1)^{d} \frac{\alpha^{\prime}}{4} e^{-2 \phi} \star H^{(0)} \wedge d A_{A}\right)\right. \\
& -\left(\frac{\alpha^{\prime}}{2} \Psi_{\chi}+\frac{\alpha^{\prime}}{4} \chi_{A} A^{A}\right) \wedge \mathbf{E}_{\exp B}^{(0)} \\
& -d\left(\frac{\alpha^{\prime}}{2} \Psi_{\chi}+\frac{\alpha^{\prime}}{4} \chi_{A} A^{A}\right) \wedge\left(e^{-2 \phi} \star H^{(0)}\right) \\
& \left.+\frac{\alpha^{\prime}}{2} e^{-2 \phi}\left(\star F_{A}-\frac{1}{2} \star H^{(1)} \wedge A_{A}\right) \wedge \mathcal{D} \chi^{A}\right\} \\
\equiv & \int d \mathbf{J}[\chi]
\end{aligned}
$$

The same arguments we made in the previous case lead to the existence of a $(d-2)$-form $\mathbf{Q}[\chi]$ such that $\mathbf{J}[\chi]=d \mathbf{Q}[\chi]$. The $(d-2)$-form is given by

$$
\mathbf{Q}[\chi]=-(-1)^{d} \frac{\alpha^{\prime}}{2}\left\{e^{-2 \phi} \star\left(-\chi^{A} F_{A}\right)+(-1)^{d} \Psi_{\chi} \wedge\left(e^{-2 \phi} \star H^{(0)}\right)\right\} .
$$

For Abelian vector fields the $\kappa^{A_{\mathrm{S}}}$ are constant and $\Psi_{\kappa}=\kappa_{A} A^{A}$ (up to a total derivative) and we recover immediately the $\mathbf{Q}[\chi]$ found in ref. [27]. On the other hand, when we change $\Psi_{\kappa}$ by a total derivative, $\mathbf{Q}[\kappa]$ is invariant on-shell up to a total derivative which will not contribute to the charge which is now given by the integral

$$
\mathcal{Q}[\kappa]=-\frac{g_{s}^{(d) 2}}{16 \pi G_{N}^{(d)}} \int_{S^{d-2}}(-1)^{d} \frac{\alpha^{\prime}}{2}\left\{e^{-2 \phi} \star d \Psi_{\kappa}+(-1)^{d} \Psi_{\kappa} \wedge\left(e^{-2 \phi} \star H^{(0)}\right)\right\},
$$

where we have made use of the definition of the vertical momentum map $\Psi_{\kappa}$ in eq. (3.17). 


\subsubsection{Lorentz charge}

Let us now consider local Lorentz transformations. As we have stressed repeatedly we can treat the local Lorentz transformations and the torsionful spin connection in parallel to the YM gauge transformations and the gauge fields. The only difference is the presence of one additional term in the Lorentz case: the Einstein-Hilbert case. If we follow the same steps as in the YM case we arrive to

$$
\mathbf{Q}[\sigma]=(-1)^{d-1} e^{-2 \phi} \star\left(e^{a} \wedge e^{b}\right) \sigma_{a b}-(-1)^{d} \frac{\alpha^{\prime}}{2}\left\{e^{-2 \phi} \star\left(-\sigma^{a}{ }_{b} R^{(0) b}{ }_{a}\right)+(-1)^{d} \Pi_{\sigma} \wedge\left(e^{-2 \phi} \star H^{(0)}\right)\right\},
$$

where $\Pi_{\sigma}$ is a 1 -form that becomes a vertical Lorentz momentum map when the Lorentz parameter $\sigma_{b}^{a}=\kappa_{b}^{a}$, a Lorentz parameter that generates a symmetry of the field configuration, i.e. a vertical Killing vector. This happens when the Vielbein and the spin connection are left invariant

$$
\begin{aligned}
& \kappa^{a}{ }_{b} e^{b}=0, \\
& \mathcal{D} \kappa^{a}{ }_{b}=0 .
\end{aligned}
$$

These two conditions imply the invariance of the torsion $\frac{1}{2} \imath_{b} \imath_{a} H^{(0)}$ Hence, they also implies the invariance of the torsionful spin connection $\Omega_{(-)}^{(0)} a$,

$$
\mathcal{D}_{(-)}^{(0)} \kappa_{b}^{a}=0
$$

These conditions can be used to modify the transformation of the KR field so that it is also left invariant, as we did in the YM case. We just quote the final form:

$$
\left.\delta_{\sigma} B=-\frac{\alpha^{\prime}}{2}\left(d \Pi_{\sigma}+\kappa_{b}^{a} R_{(-)}^{(0)} b\right)\right)-\frac{\alpha^{\prime}}{4} \mathcal{D}_{(-)}^{(0)} \sigma_{b}^{a} \wedge \Omega_{(-)}^{(0) b},
$$

where the vertical Lorentz momentum map $\Pi_{\sigma}$ is such that, when $\sigma_{b}^{a}=\kappa_{b}^{a}$

$$
d \Pi_{\kappa}=\kappa_{b}^{a} R_{(-)}^{(0) b} .
$$

The conserved charge is the integral of the $(d-2)$-form eq. (3.26) for vertical Killing vector fields $\kappa_{b}^{a}$ satisfying eqs. (3.27) and (3.27b). The first condition annihilates the first term, corresponding to the Einstein-Hilbert term in the action but the rest of the terms survive in this case and we get the non-vanishing Lorentz charge

$$
\mathcal{Q}[\kappa]=\frac{g_{s}^{(d) 2}}{16 \pi G_{N}^{(d)}} \int_{S^{d-2}}\left\{(-1)^{d} \frac{\alpha^{\prime}}{2}\left[e^{-2 \phi} \star d \Pi_{\kappa}+(-1)^{d} \Pi_{\kappa} \wedge\left(e^{-2 \phi} \star H^{(0)}\right)\right]\right\} .
$$

In the proof of the first law we will find the integral of $(d-2)$-form eq. (3.26) for a Lorentz parameter that satisfies eq. (3.27b) only. This integral give, precisely, the entropy. 


\subsection{The transformations under diffeomorphisms}

Now we turn our attention to the diffeomorphisms. Our treatment is similar to the treatment of the $\delta_{\chi}$ gauge transformations, although the use of compensating gauge transformations admits a more general justification in terms of the gauge covariance of the modified transformations (covariant Lie derivatives). Since we have discussed these modifications at length in refs. $[8,27]$ we will only discuss the aspects not covered there: torsionful spin connections, non-Abelian gauge fields and the more complicated transformations of the KR 2-form.

In this section $k$ will always be a (horizontal) Killing vector which generates a symmetry of the complete field configuration.

\subsubsection{Lie-Lorentz derivatives}

The transformations of the Vielbeins, the Levi-Civita spin connection and its curvature 2form have been discussed in refs. [8, 27], but it is convenient to adapt some of the formulae to the torsionful spin connection. They are generically given in terms of the Lie-Lorentz (or Lorentz-covariant Lie derivative refs. $[11,12,14-17]$ ) by $\delta_{\xi}=-\mathbb{L}_{\xi}$. Therefore, we will continue this discussion in terms of the latter.

The parameter of the compensating local Lorentz transformation that appears in the Lie-Lorentz derivative of $\Omega_{(-)}^{(0) a b}$ is still given by

$$
\sigma_{\xi}^{a b}=\imath_{\xi} \omega^{a b}-\nabla^{[a} \xi^{b]},
$$

but it is useful to rewrite it using $\Omega_{(-)}^{(0) a b}$ in the covariant derivatives. Due to the complete antisymmetry of the torsion, it takes the simple form

$$
\sigma_{\xi}^{a b}=\imath_{\xi} \Omega_{(-)}^{(0) a b}-\mathcal{D}_{(+)}^{(0)}\left[{ }^{[a} \xi^{b]} .\right.
$$

Observe that the presence of fully antisymmetric torsion does not modify the Killing equation $^{15}$

$$
2 \mathcal{D}_{( \pm)(a}^{(0)} \xi_{b)}=0
$$

Notice that eqs. (3.33) and (3.34) are completely independent of $H^{(0)}$ even if we have formally rewritten them in terms of the torsionful spin connection $\Omega_{(-)}^{(0)}$.

The Lie-Lorentz derivative of the torsion $\imath_{b} \imath_{a} H^{(0)}$ follows the general formula while that of the Levi-Civita connection $\omega^{a b}$ is given by

$$
\mathbb{L}_{\xi} \omega^{a b}=£_{\xi} \omega^{a b}-\mathcal{D} \sigma_{\xi}{ }^{a b},
$$

and, therefore, it is easy to see that

$$
\mathbb{L}_{\xi} \Omega_{(-)}^{(0) a b}=£_{\xi} \Omega_{(-)}^{(0) a b}-\mathcal{D}_{(-)}^{(0)} \sigma_{\xi}^{a b},
$$

and it is equally easy to see that it can be rewritten in the form

$$
\mathbb{L}_{\xi} \Omega_{(-)}^{(0) a b}=\imath_{\xi} R_{(-)}^{(0) a b}+\mathcal{D}_{(-)} P_{(-) \xi}^{a b},
$$

\footnotetext{
${ }^{15}$ The presence of generic torsion does modify the Killing equation.
} 
with

$$
P_{(-) \xi}^{a b} \equiv \mathcal{D}_{(+)}^{(0)}\left[\xi^{[a}\right.
$$

The identity

$$
\xi^{\nu} R_{(-) \nu \mu}^{(0)}{ }^{a b}+\mathcal{D}_{(-) \mu}^{(0)} P_{(-) \xi^{a b}}=\mathcal{D}_{(-)}^{(0)}\left[a\left(\nabla^{b]} \xi_{\mu}+\nabla_{\mu} \xi^{b]}\right)-\frac{3}{2} \nabla_{[\mu \mid}\left(\xi^{\nu} H_{\nu \mid \rho \sigma]}^{(0)}\right) e^{a \rho} e^{b \sigma},\right.
$$

proves that $\delta_{\xi} \Omega_{(-)}^{(0) a b}=-\mathbb{L}_{\xi} \Omega_{(-)}^{(0) a b}$ vanishes when $\xi^{\mu}=k^{\mu}$, because, in that case,

$$
-\imath_{k} R_{(-)}^{(0) a b}=\mathcal{D}_{(-)}^{(0)} P_{(-) k}^{a b} .
$$

Because $P_{(-) k}^{a b}$ satisfies this equation, we will call it the horizontal Lorentz momentum map associated to the torsionful spin connection.

$k$, then, generates a diffeomorphism that leaves the metric invariant and the KR 3-form field strength.

Again, $P_{(-) \xi}{ }^{a b}$ is a Lorentz tensor and $\delta_{\xi} \Omega_{(-)}^{(0) a b}=-\mathbb{L}_{\xi} \Omega_{(-)}^{(0) a b}$ is a Lorentz tensor, although $\Omega_{(-)}^{(0) a b}$ is a connection. When it vanishes, it vanishes in all Lorentz frames.

\subsubsection{Lie-Yang-Mills derivatives}

Since the spin connection is just the connection of the Lorentz group, this case is very similar to the previous one, the main difference being that the YM fields are fundamental fields while the spin connection is a composite field. Apart from this, in many (but not all, because of the absence of a YM analogue of the Vielbein) instances we may just apply the same formulae with the sole change of the adjoint group indices, as we are going to see.

In order to find the gauge-covariant Lie derivative of YM fields it is convenient to consider the Lie-Lorentz derivative of the curvature tensor first. In this case, since we do not know the form of the parameter of the compensating gauge transformation, we can simply consider the standard Lie derivative of the gauge field strength 2 -form defined in eq. (2.4):

$$
£_{\xi} F^{A}=\left(\imath_{\xi} d+d \imath_{\xi}\right) F^{A}=\mathcal{D} \imath_{\xi} F^{A}-f_{B C}{ }^{A} \imath_{\xi} A^{B} F^{C},
$$

where we have used the Bianchi identity $\mathcal{D} F^{A}=0$.

When $\xi=k$ this expression should vanish up to an infinitesimal gauge transformation with some parameter that we denote by $\tilde{\chi}_{k}{ }^{A}$. Then,

$$
\mathcal{D} \imath_{k} F^{A}=f_{B C}{ }^{A}\left(\imath_{k} A^{B}+\tilde{\chi}_{k}{ }^{B}\right) F^{C} \equiv f_{B C}{ }^{A} P_{k}{ }^{B} F^{C},
$$

which, upon use of the Ricci identity eq. (3.5a), can be solved by a $P_{k}{ }^{A}$ that we call the (horizontal) Yang-Mills momentum map satisfying the equation

$$
-\imath_{k} F^{A}=\mathcal{D} P_{k}{ }^{A} .
$$

Eq. (3.40) is nothing but a particular case of this equation for which the momentum map is explicitly known. This happens because we know how to express the gauge field in terms of a more fundamental field (the Vielbein). In general, the general form of $P_{k}{ }^{A}$ is 
not known but is determined up to a covariantly-constant gauge parameter. We will use a $P_{\xi}{ }^{A}$ which is undetermined except for the fact that it reduces to $P_{k}{ }^{A}$ satisfying eq. (3.43) for Killing vectors.

Now, we can use as a definition of the Lie-Yang-Mills derivative of $F^{A}$ the following expression which is guaranteed to vanish when $\xi=k$ on account of eq. (3.42):

$$
\mathbb{L}_{\xi} F^{A}=\mathcal{D} \iota_{\xi} F^{A}-f_{B C}{ }^{A} P_{\xi}^{B} F^{C}=£_{\xi} F^{A}-\delta_{\chi_{\xi}} F^{A}
$$

where the gauge compensating parameter $\chi_{\xi}{ }^{A}$ is given by the (now usual) expression

$$
\chi_{\xi}^{A}=\imath_{\xi} A^{A}-P_{\xi}^{A} .
$$

The Lie-Yang-Mills derivative of the gauge field is then

$$
\mathbb{L}_{\xi} A^{A} \equiv £_{\xi} A^{A}-\mathcal{D} \chi_{\xi}{ }^{A}=\imath_{\xi} F^{A}+\mathcal{D} P_{\xi}{ }^{A},
$$

and, by construction, it vanishes automatically when $\xi$ is a Killing vector field $k^{\mu}$ and $P_{k}{ }^{A}$ is the momentum map satisfying eq. (3.43).

\subsubsection{The Kalb-Ramond field}

The parameters of the compensating YM and local Lorentz transformations of the KR field are the same transformations $\chi_{\xi}^{A}$ and $\sigma_{\xi}^{a b}$ that we perform on other fields with YM and Lorentz indices, given by eqs. (3.45) and (3.32). Thus, if we want to construct a transformation of this field under diffeomorphisms that annihilates it when $\xi=k$ by combining its standard Lie derivative with gauge transformations, the only gauge parameter we can still play with is the 1 -form $\Lambda$ because the rest are already completely determined. We have

$$
\begin{aligned}
\delta_{\xi} B & =-£_{\xi} B+\left(\delta_{\Lambda_{\xi}}+\delta_{\chi_{\xi}}+\delta_{\sigma_{\xi}}\right) B \\
& =-£_{\xi} B+d \Lambda_{\xi}-\frac{\alpha^{\prime}}{4} \chi_{\xi} d A^{A}-\frac{\alpha^{\prime}}{4} \sigma_{\xi}{ }^{a} b d \Omega_{(-)}^{(0) b} a
\end{aligned}
$$

Again, it is convenient to start by considering the transformation of the 3-form field strength $H^{(1)}$ defined in eq. (2.6) under diffeomorphisms, because it is gauge invariant:

$$
\begin{aligned}
\delta_{\xi} H^{(1)} & =-£_{\xi} H^{(1)} \\
& =-\imath_{\xi} d H^{(1)}-d \imath_{\xi} H^{(1)} \\
& =-d_{\xi} H^{(1)}-\frac{\alpha^{\prime}}{2}\left(\imath_{\xi} F_{A} \wedge F^{A}+\imath_{\xi} R_{(-)}^{(0) a} b \wedge R_{(-)}^{(0) b}\right)
\end{aligned}
$$

where we have used the Bianchi identity eq. (2.7).

When $\xi=k$ we can use eqs. (3.40) and (3.43), integrate by parts, and use the Bianchi identities for the curvatures, getting:

$$
\begin{aligned}
\delta_{k} H^{(1)} & =-d \imath_{k} H^{(1)}+\frac{\alpha^{\prime}}{2}\left(\mathcal{D} P_{k A} \wedge F^{A}+\mathcal{D}_{(-)} P_{(-) k}^{a} b \wedge R_{(-)}^{(0) b} a\right) \\
& =-d\left[\imath_{k} H^{(1)}-\frac{\alpha^{\prime}}{2}\left(P_{k A} F^{A}+P_{(-) k b}^{a} R_{(-)}^{(0) b} a\right)\right] .
\end{aligned}
$$


By assumption, the above expression must vanish identically. Therefore, locally, there must exist a gauge-invariant 1-form, the horizontal Kalb-Ramond momentum map $P_{k}$, satisfying

$$
-\imath_{k} H^{(1)}+\frac{\alpha^{\prime}}{2}\left(P_{k A} F^{A}+P_{(-) k}^{a} b R_{(-)}^{(0)} b\right)=d P_{k} .
$$

Then, if we apply the rule of thumb that the parameter of the compensating gauge transformation is the inner product of the vector that generates the diffeomorphisms with the "connection" (here $B$ ) minus the momentum map (here some 1-form $P_{\xi}$ that in this case satisfies eq. (3.50) when $\xi=k$ )

$$
\Lambda_{\xi}=\imath_{\xi} B-P_{\xi},
$$

we arrive at the following candidate for $\delta_{\xi} B$ :

$$
\begin{aligned}
\delta_{\xi} B= & -£_{\xi} B+d \Lambda_{\xi}-\frac{\alpha^{\prime}}{4}\left(\chi_{\xi A} d A^{A}+\sigma_{\xi}^{a} b d \Omega_{(-)}^{(0) b} a\right) \\
= & -\imath_{\xi} H^{(1)}-\frac{\alpha^{\prime}}{4}\left(A_{A} \wedge \imath_{\xi} F^{A}+\Omega_{(-) b}^{(0) a} \wedge \imath_{\xi} R_{(-)}^{(0) b} a\right) \\
& -d P_{\xi}+\frac{\alpha^{\prime}}{4}\left(P_{\xi A} d A^{A}+P_{(-) \xi}^{a} b d \Omega_{(-)}^{(0) b} b\right) .
\end{aligned}
$$

Let us see if, with this definition, $\delta_{k} B=0$. Using eqs. (3.50), (3.43) and (3.40) we get, instead of zero, a total derivative

$$
\delta_{k} B=-\frac{\alpha^{\prime}}{4} d\left(P_{k A} A^{A}+P_{(-)}{ }^{a}{ }_{b} \Omega_{(-)}^{(0)} b\right),
$$

which we can simple absorb in redefinition of $\Lambda_{\xi}$ in eq. (3.51):

$$
\Lambda_{\xi} \equiv \iota_{\xi} B-P_{\xi}+\frac{\alpha^{\prime}}{4} d\left(P_{\xi A} A^{A}+P_{(-)} \xi^{a} b_{(-)}^{(0) b}\right) .
$$

With this new parameter,

$$
\begin{aligned}
\delta_{\xi} B= & -£_{\xi} B+d \Lambda_{\xi}-\frac{\alpha^{\prime}}{4} \chi_{\xi A} d A^{A}-\frac{\alpha^{\prime}}{4} \sigma_{\xi}^{a}{ }_{b} d \Omega_{(-)}^{(0) b} a \\
= & -\left[\imath_{\xi} H^{(1)}-\frac{\alpha^{\prime}}{2}\left(P_{\xi A} F^{A}+P_{(-) \xi}{ }^{a}{ }_{b} R_{(-)}^{(0) b}\right)+d P_{\xi}\right] \\
& +\frac{\alpha^{\prime}}{4}\left(A_{A} \wedge \delta_{\xi} A^{A}+\Omega_{(-)}^{(0) a}{ }_{b} \wedge \delta_{\xi} \Omega_{(-)}^{(0) b}\right) \\
\equiv & -\mathbb{L}_{\xi} B,
\end{aligned}
$$

that vanishes identically when $\xi=k$ by virtue of the definition of the KR momentum map eq. (3.50) and of $\delta_{\xi} A^{A}=\delta_{\xi} \Omega_{(-)}^{(0) b} a=0$.

The behavior of this variation under gauge transformations is far from obvious. A direct calculation gives

$$
\delta_{\text {gauge }} \delta_{\xi} B=\frac{\alpha^{\prime}}{4}\left(d \chi_{A} \wedge \delta_{\xi} A^{A}+d \sigma^{a}{ }_{b} \wedge \delta_{\xi} \Omega_{(-)}^{(0) b}\right),
$$

with $\delta_{\xi} A^{A}=-\mathbb{L}_{\xi} A^{A}$ with the Lie-Yang-Mills covariant derivative given by eq. (3.46) and with $\delta_{\xi} \Omega_{(-)}^{(0) a b}=-\mathbb{L}_{\xi} \Omega_{(-)}^{(0) a b}$, with the Lie-Lorentz derivative given by eq. (3.37). Therefore, although the $\delta_{\xi} B$ defined above is not gauge-invariant, $\delta_{k} B$ vanishes in a gaugeinvariant way. 


\subsection{The Wald-Noether charge}

Now we consider the variation of the action $S^{(1)}$ given in eq. (2.8) under the transformations $\delta_{\xi}=-\mathbb{L}_{\xi}$ for all the fields, where $\mathbb{L}_{\xi}$ is the gauge-covariant derivative, which for the Vielbein is given by $[8]$

$$
\mathbb{L}_{\xi} e^{a}=\mathcal{D} \xi^{a}+P_{\xi}{ }_{b}{ }^{b} e^{b},
$$

for the torsionful spin connection in eq. (3.37), for the YM fields in eq. (3.46) and for the KR field in eq. (3.55).

From eq. (2.13)

$$
\begin{aligned}
\delta_{\xi} S^{(1)}= & -\int\left\{\mathbf{E}_{\exp a}^{(1)} \wedge\left(\mathcal{D} \imath_{\xi} e^{a}+P_{\xi}{ }^{a} b^{b}\right)+\mathbf{E}_{\phi}^{(1)} \imath_{\xi} d \phi\right. \\
& +\mathbf{E}_{A}^{(1)} \wedge\left(\imath_{\xi} F^{A}+\mathcal{D} P_{\xi}^{A}\right)+\mathbf{E}^{(1) b}{ }_{a} \wedge\left(\imath_{\xi} R_{(-) b}^{(0) a}+\mathcal{D}_{(-)} P_{(-) \xi}{ }^{a} b\right) \\
& +\mathbf{E}_{\exp B}^{(1)} \wedge\left[\imath_{\xi} H^{(1)}+\frac{\alpha^{\prime}}{4}\left(A_{A} \wedge \imath_{\xi} F^{A}+\Omega_{(-) b}^{(0) a} \wedge \imath_{\xi} R_{(-)}^{(0) b}\right)\right. \\
& \left.-\frac{\alpha^{\prime}}{4}\left(P_{\xi A} d A^{A}+P_{(-) \xi}{ }^{a} b d \Omega_{(-)}^{(0) b}\right)+d\left[P_{\xi}-\frac{\alpha^{\prime}}{4}\left(P_{\xi A} A^{A}+P_{(-) \xi}{ }^{a} \Omega^{(0) b} \Omega_{(-)} a\right)\right]\right] \\
& \left.-d \Theta^{(1)}\left(\varphi, \delta_{\xi} \varphi\right)\right\}
\end{aligned}
$$

where $\boldsymbol{\Theta}^{(1)}\left(\varphi, \delta_{\xi} \varphi\right)$ is given by

$$
\begin{aligned}
& \boldsymbol{\Theta}^{(1)}\left(\varphi, \delta_{\xi} \varphi\right)=e^{-2 \phi} \star\left(e^{a} \wedge e^{b}\right) \wedge\left(\imath_{\xi} R_{a b}+\mathcal{D} P_{\xi a b}\right)-2 \imath_{a} d e^{-2 \phi} \star\left(e^{a} \wedge e^{b}\right) \wedge\left(\mathcal{D} \imath_{\xi} e_{b}+P_{\xi b c} e^{c}\right) \\
& +8 e^{-2 \phi} \star d \phi \imath_{\xi} d \phi \\
& -e^{-2 \phi} \star H^{(1)} \wedge\left\{\imath_{\xi} H^{(1)}+\frac{\alpha^{\prime}}{4}\left(A_{A} \wedge \imath_{\xi} F^{A}+\Omega_{(-)}^{(0) a} b \wedge \imath_{\xi} R_{(-)}^{(0) b}\right)\right. \\
& \left.-\frac{\alpha^{\prime}}{4}\left(P_{\xi A} d A^{A}+P_{(-)} \xi_{b}^{a} d \Omega_{(-)}^{(0) b}\right)+d\left[P_{\xi}-\frac{\alpha^{\prime}}{4}\left(P_{\xi A} A^{A}+P_{(-)} \xi_{b}^{a} \Omega_{(-)}^{(0) b}\right)\right]\right\} \\
& -\frac{\alpha^{\prime}}{2} e^{-2 \phi}\left(\star F_{A}-\frac{1}{2} \star H^{(0)} \wedge A_{A}\right) \wedge\left(\imath_{\xi} F^{A}+\mathcal{D} P_{\xi}{ }^{A}\right) . \\
& -\frac{\alpha^{\prime}}{2} e^{-2 \phi}\left(\star R_{(-)}^{(0) b}{ }_{a}-\frac{1}{2} \star H^{(0)} \wedge \Omega_{(-)}^{(0) b}\right) \wedge\left(\imath_{\xi} R_{(-)}^{(0) a} b+\mathcal{D}_{(-)} P_{(-) \xi}{ }^{a} b\right) .
\end{aligned}
$$

Integrating by parts and using the Noether identities eqs. (3.6a), (3.6b), (3.6c), (3.7) and the Noether identity associated to the invariance under diffeomorphisms

$$
\begin{aligned}
& (-1)^{d} \mathcal{D} \mathbf{E}_{\exp a}^{(1)} \imath_{\xi} e^{a}+\mathbf{E}_{\exp B}^{(1)} \wedge \imath_{\xi} H^{(1)}+\mathbf{E}_{\phi}^{(1)} \imath_{\xi} d \phi \\
& +\left(\mathbf{E}_{A}^{(1)}+\frac{\alpha^{\prime}}{4} \mathbf{E}_{\exp B}^{(0)} \wedge A_{A}\right) \wedge \imath_{\xi} F^{A}+\left(\mathbf{E}^{(1) b}{ }_{a}+\frac{\alpha^{\prime}}{4} \mathbf{E}_{\exp B}^{(0)} \wedge \Omega_{(-)}^{(0) b}\right) \wedge \imath_{\xi} R_{(-)}^{(0) a}{ }_{b}=0
\end{aligned}
$$

we can see that the volume term in the variation of the action eq. (3.58) reduces to another total derivative

$$
\delta_{\xi} S^{(1)}=\int d \Theta^{(1) \prime}\left(\varphi, \delta_{\xi} \varphi\right)
$$


with

$$
\begin{aligned}
\Theta^{(1) \prime}\left(\varphi, \delta_{\xi} \varphi\right)= & \Theta^{(1)}\left(\varphi, \delta_{\xi} \varphi\right) \\
& +(-1)^{d} \mathbf{E}_{\exp a}^{(1)} e^{a}+(-1)^{d-1} \mathbf{E}_{\exp B}^{(1)} \wedge P_{\xi} \\
& +(-1)^{d}\left(\mathbf{E}_{A}^{(1)}+\frac{\alpha^{\prime}}{4} \mathbf{E}_{\exp B}^{(0)} \wedge A_{A}\right) P_{\xi} A \\
& +(-1)^{d}\left(\mathbf{E}^{(1) b}{ }_{a}+\frac{\alpha^{\prime}}{4} \mathbf{E}_{\exp B}^{(0)} \wedge \Omega_{(-)}^{(0) b}\right) P_{(-) \xi}{ }^{a} b .
\end{aligned}
$$

The usual reasoning leads us to the off-shell identity

$$
d \mathbf{J}^{(1)}[\xi]=0,
$$

where

$$
\mathbf{J}^{(1)}[\xi] \equiv \boldsymbol{\Theta}^{(1) \prime}\left(\varphi, \delta_{\xi} \varphi\right)+\imath_{\xi} \mathbf{L}^{(1)},
$$

and to the local existence of a $(d-2)$-form $\mathbf{Q}^{(1)}[\xi]$ such that $\mathbf{J}^{(1)}[\xi]=d \mathbf{Q}^{(1)}[\xi]$.

A straightforward calculation leads to the fully gauge-invariant Wald-Noether charge

$$
\begin{aligned}
\mathbf{Q}^{(1)}[\xi]= & (-1)^{d} \star\left(e^{a} \wedge e^{b}\right)\left[e^{-2 \phi} P_{\xi a b}-2 \imath_{a} d e^{-2 \phi} \xi_{b}\right] \\
& +(-1)^{d-1} \frac{\alpha^{\prime}}{2}\left[P_{\xi A} e^{-2 \phi} \star F^{A}+P_{(-) \xi^{a} b}\left(e^{-2 \phi} \star R_{(-)}^{(0) b}\right)\right] \\
& -P_{\xi} \wedge\left(e^{-2 \phi} \star H^{(1)}\right),
\end{aligned}
$$

which is one of the main results of this paper.

\section{Restricted generalized zeroth laws}

One of the main ingredients in Wald's approach to the first law of black hole mechanics is the zeroth law stating that $\kappa$ is constant over the horizon [36]. Originally, this law was proved using the Einstein equations and the dominant energy condition (see, for instance, ref. [42]) but a completely geometrical proof was presented in ref. [43].

In presence of an electromagnetic field one also needs to use the generalized zeroth law that guarantees that the electrostatic potential is also constant over the whole horizon. There is no purely geometrical proof of this law, though, and the standard proof also makes use of the Einstein equations and of the dominant energy condition. In ref. [27] we have explained how this proof can be extended to a theory containing an arbitrary number of Abelian vector fields and the KR field coupled to them via Chern-Simons terms. Essentially one gets a sum of non-negative terms containing the contribution of each field, and each of them has to vanish. Extending this proof to the non-Abelian case is possible, as long as we restrict ourselves to a gauge group with definite positive Killing metric because one gets sums of non-negative terms. However, the $R_{(-)}^{(0) 2}$ term of our theory is of YM type, but with non-definite Killing metric because of the non-compactness of the Lorentz group and the proof cannot be extended to this case in a straightforward manner.

It is, however, possible to prove the first law in bifurcate horizons if one can proof generalized zeroth laws for the matter fields restricted to the bifurcation sphere $\mathcal{B H}$ where the 
Killing vector associated to the event horizon, $k$, vanishes identically. These restricted generalized zeroth laws state the closedness of certain differential forms on $\mathcal{B H}$. The definitions of the potentials as certain constants follow from them as we are going to explain.

Assuming all the fields are regular over the horizon, it is clear that the inner products of their field strengths with $k$ must vanish on $\mathcal{B H}$ :

$$
\begin{aligned}
& \imath_{k} d \phi \stackrel{\mathcal{B H}}{=} 0, \\
& \imath_{k} H \stackrel{\mathcal{B H}}{=} 0, \\
& \imath_{k} F^{A} \stackrel{\mathcal{B H}}{=} 0, \\
& \imath_{k} R_{(-)}^{(0) a} \stackrel{\stackrel{\mathcal{B H}}{=}}{=} 0 .
\end{aligned}
$$

Eq. (4.1a) is actually true over the whole spacetime, by assumption. From eq. (4.1c) and the definition of the YM momentum map $P_{k}{ }^{A}$ we find that

$$
\mathcal{D} P_{k}^{A} \stackrel{\mathcal{B H}}{=} 0
$$

which tells us that the horizontal YM momentum map $P_{k}{ }^{A}$ is, at the same time, a vertical Killing vector field on $\mathcal{B H}$. This is what is needed in order to have an associated conserved charge there (see the discussion in section 3.2.2).

Analogously, from eq. (4.1d) and the definition of the momentum map $P_{(-) k}{ }_{b}$ eq. (3.40) we get

$$
\mathcal{D}_{(-)}^{(0)} P_{(-) k} a \stackrel{\mathcal{B H}}{=} 0
$$

which tells us that the horizontal Lorentz momentum map $P_{k}{ }^{A}$ is, also, a vertical Killing vector field on $\mathcal{B H}$.

Observe that the last two equations have as a consequence the existence of the gaugeinvariant 1-forms $\Psi_{P_{k}}$ and $\Pi_{P_{k}}$ defined by

$$
\begin{aligned}
& d \Pi_{P_{k}} \stackrel{\stackrel{\mathcal{B H}}{=} P_{(-) k}{ }^{a}{ }_{b} R_{(-)}^{(0) b} a}{ }, \\
& d \Psi_{P_{k}} \stackrel{\mathcal{B H}}{=} P_{k A} F^{A} .
\end{aligned}
$$

The closedness of the right-hand sides of these equations on $\mathcal{B H}$, which guarantee the local existence of $\Psi_{P_{k}}$ and $\Pi_{P_{k}}$ there are the restricted generalized zeroth laws for the YM and torsionful spin connection fields.

Finally, from eq. (4.1b) and the definition of the KR momentum map eq. (3.50) plus the above two equations that define $\Psi_{P_{k}}$ and $\Pi_{P_{k}}$ we get

$$
d\left[P_{k}-\frac{\alpha^{\prime}}{2}\left(\Psi_{P_{k}}+\Pi_{P_{k}}\right)\right] \stackrel{\mathcal{B H}}{=} 0,
$$

which is the restricted generalized zeroth law of the KR field. 


\section{The first law}

Following Wald [5], we start by defining the pre-symplectic $(d-1)$-form [4]

$$
\omega^{(1)}\left(\varphi, \delta_{1} \varphi, \delta_{2} \varphi\right) \equiv \delta_{1} \Theta^{(1)}\left(\varphi, \delta_{2} \varphi\right)-\delta_{2} \Theta^{(1)}\left(\varphi, \delta_{1} \varphi\right),
$$

and the symplectic form relative to the Cauchy surface $\Sigma$

$$
\Omega^{(1)}\left(\varphi, \delta_{1} \varphi, \delta_{2} \varphi\right) \equiv \int_{\Sigma} \omega^{(1)}\left(\varphi, \delta_{1} \varphi, \delta_{2} \varphi\right)
$$

When $\varphi$ is a solution of the equations of motion $\mathbf{E}_{\varphi}=0, \delta_{1} \varphi=\delta \varphi$ is an arbitrary variation of the fields and $\delta_{2} \varphi=\delta_{\xi} \varphi$ is their variation under diffeomorphisms [6]

$$
\omega^{(1)}\left(\varphi, \delta \varphi, \delta_{\xi} \varphi\right)=\delta \mathbf{J}^{(1)}+d l_{\xi} \boldsymbol{\Theta}^{(1) \prime}=\delta d \mathbf{Q}^{(1)}[\xi]+d \imath_{\xi} \boldsymbol{\Theta}^{(1) \prime},
$$

where, in our case, the Noether-Wald $(d-2)$-form charge $\mathbf{Q}^{(1)}$ is given by eq. (3.65) and $\boldsymbol{\Theta}^{\prime}$ is given in eq. (3.62). Since, on-shell, $\boldsymbol{\Theta}^{(1)}=\boldsymbol{\Theta}^{(1) \prime}$, we have that, if $\delta \varphi$ satisfies the linearized equations of motion, $\delta d \mathbf{Q}^{(1)}=d \delta \mathbf{Q}^{(1)}$. Furthermore, if the parameter $\xi=k$ generates a transformation that leaves the field configuration invariant, $\delta_{k} \varphi=0,{ }^{16}$ linearity implies that $\omega^{(1)}\left(\varphi, \delta \varphi, \delta_{k} \varphi\right)=0$, and

$$
d\left(\delta \mathbf{Q}^{(1)}[k]+\imath_{k} \mathbf{\Theta}^{(1) \prime}\right)=0 .
$$

Integrating this expression over a hypersurface $\Sigma$ with boundary $\delta \Sigma$ and using Stokes' theorem we arrive at

$$
\int_{\delta \Sigma}\left(\delta \mathbf{Q}^{(1)}[k]+\imath_{k} \mathbf{\Theta}^{(1) \prime}\right)=0 .
$$

We consider field configurations that describe asymptotically flat, stationary, blackhole spacetimes with bifurcate horizons $\mathcal{H}$ and the Killing vector $k$ is the one whose Killing horizon is the black hole's event horizon. $k$, then, will be given by a linear combination with constant coefficients $\Omega^{n}$ of the timelike Killing vector associated to stationarity, $t^{\mu} \partial_{\mu}$ and the $\left[\frac{1}{2}(d-1)\right]$ generators of inequivalent rotations in $d$ spacetime dimensions $\phi_{n}^{\mu} \partial_{\mu}$

$$
k^{\mu}=t^{\mu}+\Omega^{n} \phi_{n}^{\mu} .
$$

The constant coefficients $\Omega^{n}$ are the angular velocities of the horizon.

The hypersurface $\Sigma$ to be the space bounded by infinity and the bifurcation sphere $\mathcal{B H}$ on which $k=0$, so $\delta \Sigma$ has two disconnected pieces: a $(d-2)$-sphere at infinity, $\mathrm{S}_{\infty}^{d-2}$, and the bifurcation sphere $\mathcal{B H}$. Then, taking into account that $k=0$ on $\mathcal{B H}$, we obtain the relation

$$
\delta \int_{\mathcal{B H}} \mathbf{Q}^{(1)}[k]=\int_{\mathbf{S}_{\infty}^{d-2}}\left(\delta \mathbf{Q}^{(1)}[k]+\imath_{k} \mathbf{\Theta}^{(1) \prime}\right) .
$$

As explained in refs. $[6,38]$, the right-hand side can be identified with $\delta M-\Omega^{m} \delta J_{n}$, where $M$ is the total mass of the black-hole spacetime and $J_{n}$ are the independent components of the angular momentum. ${ }^{17}$

\footnotetext{
${ }^{16}$ Notice that our goal in section 3.3 was, precisely, to construct variations of the fields $\delta_{\xi}$ with that property.

${ }^{17}$ When the spacetime has compact dimensions, the $d$-dimensional mass $M$ is a combination of the lowerdimensional mass and Kaluza-Klein charges. The details depend on the compactification and will be studied elsewhere.
} 
Using the explicit form of $\mathbf{Q}^{(1)}[k]$, eq. (3.65), noticing that $-2 \imath_{a} d e^{-2 \phi} k_{b} \stackrel{\mathcal{B H}}{=} 0$ and restoring the overall factor $g_{s}^{(d) 2}\left(16 \pi G_{N}^{(d)}\right)^{-1}$, we find

$$
\begin{aligned}
\delta \int_{\mathcal{B H}} \mathbf{Q}^{(1)}[k]= & \frac{g_{s}^{(d) 2}}{16 \pi G_{N}^{(d)}} \delta \int_{\mathcal{B H}}(-1)^{d} e^{-2 \phi} \star\left(e^{a} \wedge e^{b}\right) P_{k a b} \\
& +\frac{g_{s}^{(d) 2}}{16 \pi G_{N}^{(d)}} \delta \int_{\mathcal{B H}}(-1)^{d-1} \frac{\alpha^{\prime}}{2} P_{(-) k}{ }^{a} b\left(e^{-2 \phi} \star R_{(-)}^{(0) b} a\right) \\
& +\frac{g_{s}^{(d) 2}}{16 \pi G_{N}^{(d)}} \delta \int_{\mathcal{B H}}(-1)^{d-1} \frac{\alpha^{\prime}}{2} P_{k} e^{-2 \phi} \star F^{A} \\
& -\frac{g_{s}^{(d) 2}}{16 \pi G_{N}^{(d)}} \delta \int_{\mathcal{B H}} P_{k} \wedge\left(e^{-2 \phi} \star H^{(1)}\right) .
\end{aligned}
$$

The right-hand side of this identity is expected to be of the form $T \delta S+\Phi \delta \mathcal{Q}$ for some charges $\mathcal{Q}$ and potentials $\Phi$. However, when we compare the third and fourth integrals in the right-hand side with the definitions of the YM and KR charges eqs. (3.25) and (3.12) we see that some terms are missing in the integrand of the first and that, in the second, there is no closed or harmonic form in the integrand, since the horizontal KR momentum map is not necessarily closed on $\mathcal{B H}$. We found a similar problem in ref. [27] and the solution is essentially the same: add and subtract the same term in different integrals in order to complete the integrand of the definition of YM charge and in order to construct a 1 -form which is closed in $\mathcal{B H}$.

The 1-form which is closed on $\mathcal{B H}$ and which contains $P_{k}$ follows from the restricted generalized zeroth law of the KR field, eq. (4.5). We must add a term $-\frac{\alpha^{\prime}}{2} \Psi_{P_{k}}$ to the fourth integral and subtract the same term to the third, which now contains all the terms associated to the YM charge because of the restricted generalized zeroth law eq. (4.2). However, eq. (4.5) also tells us to add another term $-\frac{\alpha^{\prime}}{2} \Pi_{P_{k}}$ to the fourth integral and we can only compensate by subtracting it to the second. This completes the closed 1form in the fourth integral and completes the integrand of the Lorentz charge according to eq. (3.31) and thanks to the restricted generalized zeroth law eq. (4.3).

The result of these additions and subtractions is

$$
\begin{aligned}
\delta \int_{\mathcal{B H}} \mathbf{Q}^{(1)}[k]= & \frac{g_{s}^{(d) 2}}{16 \pi G_{N}^{(d)}} \delta \int_{\mathcal{B H}}(-1)^{d} e^{-2 \phi} \star\left(e^{a} \wedge e^{b}\right) P_{k a b} \\
& +\frac{g_{s}^{(d) 2}}{16 \pi G_{N}^{(d)}} \delta \int_{\mathcal{B H}}(-1)^{d-1} \frac{\alpha^{\prime}}{2}\left[e^{-2 \phi} \star d \Pi_{P_{k}}+(-1)^{d} \Pi_{P_{k}} \wedge\left(e^{-2 \phi} \star H^{(0)}\right)\right] \\
& +\frac{g_{s}^{(d) 2}}{16 \pi G_{N}^{(d)}} \delta \int_{\mathcal{B H}}(-1)^{d-1} \frac{\alpha^{\prime}}{2}\left[e^{-2 \phi} \star d \Psi_{P_{k}}+(-1)^{d} \Psi_{P_{k}} \wedge\left(e^{-2 \phi} \star H^{(0)}\right)\right] \\
& -\frac{g_{s}^{(d) 2}}{16 \pi G_{N}^{(d)}} \delta \int_{\mathcal{B H}}\left[P_{k}-\frac{\alpha^{\prime}}{2}\left(\Psi_{P_{k}}+\Pi_{P_{k}}\right)\right] \wedge\left(e^{-2 \phi} \star H^{(1)}\right)
\end{aligned}
$$


where $\Psi_{P_{k}}$ and $\Pi_{P_{k}}$ satisfy eqs. (4.4b) and (4.4a), respectively, whose integrability is guaranteed by the fact that the YM and Lorentz momentum maps are covariantly constant on $\mathcal{B H}$ (the restricted generalized zeroth laws).

Now, let us assume that the particular field configuration under consideration admits a set of covariantly constant YM parameters on $\mathcal{B H}$ that we label with an index $I, \kappa_{I}{ }^{A}$

$$
\mathcal{D} \kappa_{I}^{A} \stackrel{\mathcal{B H}}{=} 0, \quad \Rightarrow \quad P_{k} \stackrel{A}{\stackrel{\mathcal{B H}}{=}} \Phi^{I} \kappa_{I}^{A},
$$

where the constants $\Phi^{I}$ will be interpreted as the potentials associated to the YM charges $\mathcal{Q}_{I}$ computed with the parameter $\kappa_{I}{ }^{A}$ eq. (3.25)

$$
\mathcal{Q}_{I} \equiv \mathcal{Q}\left[\kappa_{I}\right]=\frac{g_{s}^{(d) 2}}{16 \pi G_{N}^{(d)}} \int_{\mathcal{B H}}(-1)^{d-1} \frac{\alpha^{\prime}}{2}\left[e^{-2 \phi} \star d \Psi_{I}+(-1)^{d} \Psi_{I} \wedge\left(e^{-2 \phi} \star H^{(0)}\right)\right],
$$

where

$$
d \Psi_{I}=-\kappa_{I A} F^{A} .
$$

As a result, the third line in eq. (5.9) becomes $\Phi^{I} \delta \mathcal{Q}_{I}$.

Now, following refs. [37, 38], as a consequence of the KR restricted generalized zeroth law eq. (4.5), we can write (Hodge decomposition)

$$
P_{k}-\frac{\alpha^{\prime}}{2}\left(\Psi_{P_{k}}+\Pi_{P_{k}}\right) \stackrel{\mathcal{B H}}{=} d e+\Phi^{i} \Lambda_{h i}
$$

where $e$ is some function, the $\Lambda_{h i}$ are the harmonic 1-forms of the bifurcation sphere and the $\Phi^{i}$ are constants that can be interpreted as the potentials associated to the KR charges $\mathcal{Q}_{i}=\mathcal{Q}\left(\Lambda_{h i}\right)$ eq. $(3.12)$

$$
\mathcal{Q}_{i}=-\frac{g_{s}^{(d) 2}}{16 \pi G_{N}^{(d)}} \int_{C_{\Lambda_{h i}}} e^{-2 \phi} \star H,
$$

where $C_{\Lambda_{h i}}$ is the $(d-3)$-cycle dual to the harmonic 1 -form $\Lambda_{h i}$ in $\mathcal{B H}$.

As a result, the fourth line in eq. (5.9) becomes $\Phi^{i} \delta \mathcal{Q}_{i}$, and we are left with the first two, which are linear in the Lorentz momentum map $P_{k}{ }^{a b}$, which, on $\mathcal{B H}$, is given by $\kappa n^{a b}$, where $n^{a b}$ is the binormal to the horizon. The terms in those two lines must, therefore, be interpreted as those giving rise to the term $T \delta S$ in the first law

$$
\delta M=T \delta S+\Phi^{I} \delta \mathcal{Q}_{I}+\Phi^{i} \delta \mathcal{Q}_{i}+\Omega^{n} \delta J_{n} .
$$

\section{Wald entropy}

It follows from the results of the previous section that the entropy is given by

$$
S=(-1)^{d} \frac{g_{s}^{(d) 2}}{8 G_{N}^{(d)}} \int_{\mathcal{B H}} e^{-2 \phi}\left\{\left[\star\left(e^{a} \wedge e^{b}\right)+\frac{\alpha^{\prime}}{2} \star R_{(-)}^{(0)} a b\right] n_{a b}+(-1)^{d} \frac{\alpha^{\prime}}{2} \Pi_{n} \wedge \star H^{(0)}\right\},
$$

where we have defined the 1 -form $\Pi_{n}$ (vertical Lorentz momentum map associated to the binormal) on the bifurcation sphere

$$
d \Pi_{n} \stackrel{\mathcal{B H}}{=} R_{(-)}^{(0) a b} n_{a b} .
$$


This is the main result of this paper, which we will discuss in the next section. It is worth stressing that the term that involves $\Pi_{n}$, and which has been shown to give an important contribution to the entropy of well-known black-hole solutions refs. [7, 9, 33-35] occurs in the entropy formula just to cancel an equivalent term that we had to add to get the correct definition of the KR charge and the associated potential. Without a detailed knowledge of the conserved charges, the restricted generalized zeroth laws and the potentials associated, the presence of that term in the entropy formula could not have been guessed.

\section{Discussion}

In this paper we have derived an entropy formula for the black-hole solutions of the Heterotic Superstring effective action to first order in $\alpha^{\prime}$ using Wald's formalism $[4,5]$ taking carefully into account all the symmetries of the theory. As a result, our entropy formula eq. (6.1) is manifestly gauge invariant. In particular, it is manifestly invariant under local Lorentz transformations.

It is interesting to compare this result with the one that would follow from the direct (and naive) application of the Iyer-Wald prescription [6]. The first two terms in eq. (6.1) can be obtained from eq. (2.8) by varying the Einstein-Hilbert term and the $R_{(-)}^{2}$ term with respect to the Riemann curvature tensor, but the third term cannot be obtained in that way from the $H^{2}$ term. As stressed in refs. [7, 9, 33], the variation of this term with respect to the Riemann tensor gives a term of the form

$$
\frac{\alpha^{\prime}}{4} e^{-2 \phi}\left(\Omega_{(-)}^{(0) a b} n_{a b}\right) \wedge \star H^{(0)},
$$

which is not Lorentz-covariant. The coefficient of this term differs from the last term in eq. (6.1) if we associate $\Pi_{n}$ to $\Omega_{(-)}^{(0)}{ }^{a b} n_{a b}$, which is the right thing to do as we are going to show. But this coefficient changes after dimensional reduction, as observed in ref. [2]. The explicit calculation in ref. [33] shows that the right coefficient is the one that arises after dimensional reduction, ${ }^{18}$ but, certainly, there are ambiguities in the way in which the Chern-Simons terms are defined in lower dimensions.

It is interesting to observe that because $\mathcal{D} n_{a b} \stackrel{\mathcal{B H}}{=} 0$,

$$
d \Pi_{n} \stackrel{\mathcal{B H}}{=} d\left(\Omega_{(-)}^{(0) a b} n_{a b}\right)+\Omega_{(-)}^{(0) a} c \wedge \Omega_{(-)}^{(0) c b} n_{a b} .
$$

For the non-extremal Reissner-Nordström black hole of ref. [44], whose $\alpha^{\prime}$ corrections were computed in ref. [33], the second term vanishes identically in the tangent space basis used (see appendix C). This shows that, in that basis, our entropy formula and the entropy formula obtained via the Iyer-Wald prescription (after dimensional reduction) give the same result. Of course, our formula is valid in any basis.

Our entropy formula seems to differ from the entropy formula obtained in ref. [45], but a detailed comparison is not possible since that formula contains undetermined parameters

\footnotetext{
${ }^{18}$ The entropy calculated in this way satisfies the first law or, equivalently, the thermodynamic relation $\frac{\partial S}{\partial M}=\frac{1}{T}$.
} 
that guarantee its invariance under Lorentz transformations. In ref. [45] it was argued that those undetermined parameters do not contribute to the entropy in certain cases but, without an explicit expression, it is difficult to understand why or when this may happen. Furthermore, as we have shown, the identification of the entropy formula can only be made after the first law of black hole mechanics has been proven, and this requires a careful identification of the conserved charges of the theory: some terms (the one involving $\Pi_{n}$ ) occur in the entropy formula only because they are needed to compensate other terms that have to appear in the correct definition of the KR charge. This analysis was simply not carried out in ref. [45].

Our entropy formula (the contribution due to the presence of Lorentz- or gravitational Chern-Simons terms in $\left.H^{(1)}\right)$ also differs from the one found in ref. [39]. Observe that eq. (40) in ref. [39], similar to the terms contained in the formulae derived in refs. [7,9] and to eq. (7.1) is not covariant. Thus, it may only give the right result in certain basis, if at all. ${ }^{19}$ The problems in the derivation of ref. [39] are having overlooked the KR conserved charge and the determination of the gauge parameters that generate symmetries of the complete field configuration.

Finally, it is interesting to note that the entropy formula looks like the charge associated to the Lorentz transformations generated by the binormal to the horizon. These transformations preserve the connections $\omega$ and $\Omega_{(-)}^{(0)}$ on the bifurcation sphere, but they do not preserve the Vielbein, as we assumed in section 3.2.3 (eq. (3.27)), which produces an additional term associated to the Einstein-Hilbert term.

The main use of the entropy formula that we have found is to put in solid ground the calculations of the macroscopic entropies of $\alpha^{\prime}$-corrected black holes, an ineluctable condition for a fair comparison with the microscopic ones. More $\alpha^{\prime}$-corrected solutions will be available to this end [47]. As mentioned in the introduction, another necessary ingredient for this comparison is the correct identification of the relation between the charges of the black hole and the branes in the string background. These results and those of our previous work [27] single out a very precise definition of the conserved charges, which turn out to be of Page type, conserved and gauge-invariant under the assumptions made. This fact should shed light on this problem and we intend to pursue this line of research in future work.

\section{Acknowledgments}

TO would like to thank G. Barnich, P. Cano, P. Meessen, P.F. Ramírez, A. Ruipérez and C. Shahbazi for many useful conversations and their long-term collaboration in this research topic. DP would also like to thank G. Barnich for many useful conversations. This work has been supported in part by the MCIU, AEI, FEDER (UE) grant PGC2018-095205-BI00 and by the Spanish Research Agency (Agencia Estatal de Investigación) through the grant IFT Centro de ExcelenciThe work of ZE has also received funding from "La Caixa" Foundation (ID 100010434), under the agreement LCF/BQ/DI18/11660042a Severo Ochoa SEV-2016-0597. TO wishes to thank M.M. Fernández for her permanent support.

\footnotetext{
${ }^{19}$ The non-covariance of Tachikawa's entropy formula was observed in ref. [46], where an alternative method was devised to deal with this problem. Nevertheless, the formula obtained in ref. [46] reduces to Tachikawa's in $\mathcal{B H}$, apparently losing the covariance, while ours does not.
} 
Open Access. This article is distributed under the terms of the Creative Commons Attribution License (CC-BY 4.0), which permits any use, distribution and reproduction in any medium, provided the original author(s) and source are credited.

\section{References}

[1] A. Strominger and C. Vafa, Microscopic origin of the Bekenstein-Hawking entropy, Phys. Lett. B 379 (1996) 99 [hep-th/9601029] [inSPIRE].

[2] F. Faedo and P.F. Ramirez, Exact charges from heterotic black holes, JHEP 10 (2019) 033 [arXiv: 1906.12287] [INSPIRE].

[3] E.A. Bergshoeff and M. de Roo, The Quartic Effective Action of the Heterotic String and Supersymmetry, Nucl. Phys. B 328 (1989) 439 [INSPIRE].

[4] J. Lee and R.M. Wald, Local symmetries and constraints, J. Math. Phys. 31 (1990) 725 [INSPIRE].

[5] R.M. Wald, Black hole entropy is the Noether charge, Phys. Rev. D 48 (1993) R3427 [gr-qc/9307038] [INSPIRE].

[6] V. Iyer and R.M. Wald, Some properties of Noether charge and a proposal for dynamical black hole entropy, Phys. Rev. D 50 (1994) 846 [gr-qc/9403028] [InSPIRE].

[7] Z. Elgood and T. Ortín, T duality and Wald entropy formula in the Heterotic Superstring effective action at first-order in $\alpha^{\prime}$, JHEP 10 (2020) 097 [arXiv:2005.11272] [INSPIRE].

[8] Z. Elgood, P. Meessen and T. Ortín, The first law of black hole mechanics in the Einstein-Maxwell theory revisited, JHEP 09 (2020) 026 [arXiv: 2006. 02792] [INSPIRE].

[9] T. Ortín, $O(n, n)$ invariance and Wald entropy formula in the Heterotic Superstring effective action at first order in $\alpha^{\prime}$, JHEP 01 (2021) 187 [arXiv:2005.14618] [INSPIRE].

[10] T. Jacobson and A. Mohd, Black hole entropy and Lorentz-diffeomorphism Noether charge, Phys. Rev. D 92 (2015) 124010 [arXiv:1507.01054] [INSPIRE].

[11] T. Ortín, A Note on Lie-Lorentz derivatives, Class. Quant. Grav. 19 (2002) L143 [hep-th/0206159] [INSPIRE].

[12] T. Ortín, Gravity and Strings, Cambridge Monographs on Mathematical Physics, Cambridge University Press, 2nd edition (2015) [DOI] [INSPIRE].

[13] L. Fatibene and M. Francaviglia, General theory of Lie derivatives for Lorentz tensors, Commun. Math 19 (2011) 11 [arXiv:0904.0258].

[14] A. Lichnerowicz, Spineurs harmoniques, C. R. Acad. Sci. Paris 257 (1963) 7.

[15] Y. Kosmann, Dérivées de Lie des spineurs, C. R. Acad. Sci. Paris Sér. A 262 (1966) A289.

[16] Y. Kosmann, Dérivées de Lie des spineurs, Annali Mat. Pura Appl. (IV) 91 (1972) 317.

[17] D.J. Hurley and M.A. Vandyck, On the concepts of Lie and covariant derivatives of spinors. Part 1, J. Phys. A 27 (1994) 4569 [inSPIRE].

[18] M.A.J. Vandyck, On the problem of space-time symmetries in the theory of supergravity, Gen. Rel. Grav. 20 (1988) 261 [INSPIRE].

[19] M.A. Vandyck, On the problem of space-time symmetries in the theory of supergravity. 2: $N=2$ supergravity and spinorial lie derivatives, Gen. Rel. Grav. 20 (1988) 905 [InSPIRE]. 
[20] J.M. Figueroa-O'Farrill, On the supersymmetries of Anti-de Sitter vacua, Class. Quant. Grav. 16 (1999) 2043 [hep-th/9902066] [INSPIRE].

[21] P.B. Aneesh, S. Chakraborty, S.J. Hoque and A. Virmani, First law of black hole mechanics with fermions, Class. Quant. Grav. 37 (2020) 205014 [arXiv:2004.10215] [INSPIRE].

[22] K. Prabhu, The First Law of Black Hole Mechanics for Fields with Internal Gauge Freedom, Class. Quant. Grav. 34 (2017) 035011 [arXiv:1511.00388] [INSPIRE].

[23] K. Hajian and M.M. Sheikh-Jabbari, Solution Phase Space and Conserved Charges: A General Formulation for Charges Associated with Exact Symmetries, Phys. Rev. D 93 (2016) 044074 [arXiv: 1512.05584] [INSPIRE].

[24] I.A. Bandos and T. Ortín, On the dualization of scalars into $(d-2)$-forms in supergravity. Momentum maps, R-symmetry and gauged supergravity, JHEP 08 (2016) 135 [arXiv: 1605.05559] [INSPIRE].

[25] E. Frodden and D. Hidalgo, Surface Charges for Gravity and Electromagnetism in the First Order Formalism, Class. Quant. Grav. 35 (2018) 035002 [arXiv:1703.10120] [INSPIRE].

[26] E. Frodden and D. Hidalgo, Surface Charges Toolkit for Gravity, Int. J. Mod. Phys. D 29 (2020) 2050040 [arXiv: 1911.07264] [INSPIRE].

[27] Z. Elgood, D. Mitsios, T. Ortín and D. Pereñíguez, The first law of heterotic stringy black hole mechanics at zeroth order in $\alpha^{\prime}$, arXiv:2012.13323 [INSPIRE].

[28] H. Nicolai and P.K. Townsend, $N=3$ Supersymmetry Multiplets with Vanishing Trace Anomaly: Building Blocks of the $N>3$ Supergravities, Phys. Lett. B 98 (1981) 257 [INSPIRE].

[29] T. Regge and C. Teitelboim, Role of Surface Integrals in the Hamiltonian Formulation of General Relativity, Annals Phys. 88 (1974) 286 [INSPIRE].

[30] L.F. Abbott and S. Deser, Stability of Gravity with a Cosmological Constant, Nucl. Phys. B 195 (1982) 76 [INSPIRE].

[31] G. Barnich and F. Brandt, Covariant theory of asymptotic symmetries, conservation laws and central charges, Nucl. Phys. B 633 (2002) 3 [hep-th/0111246] [INSPIRE].

[32] G. Barnich, Boundary charges in gauge theories: Using Stokes theorem in the bulk, Class. Quant. Grav. 20 (2003) 3685 [hep-th/0301039] [INSPIRE].

[33] P.A. Cano, S. Chimento, R. Linares, T. Ortín and P.F. Ramírez, $\alpha^{\prime}$ corrections of Reissner-Nordström black holes, JHEP 02 (2020) 031 [arXiv:1910.14324] [INSPIRE].

[34] P.A. Cano, P. Meessen, T. Ortín and P.F. Ramírez, $\alpha^{\prime}$-corrected black holes in String Theory, JHEP 05 (2018) 110 [arXiv:1803.01919] [INSPIRE].

[35] P.A. Cano, S. Chimento, P. Meessen, T. Ortín, P.F. Ramírez and A. Ruipérez, Beyond the near-horizon limit: Stringy corrections to Heterotic Black Holes, JHEP 02 (2019) 192 [arXiv: 1808.03651] [INSPIRE].

[36] J.M. Bardeen, B. Carter and S.W. Hawking, The Four laws of black hole mechanics, Commun. Math. Phys. 31 (1973) 161 [inSPIRE].

[37] K. Copsey and G.T. Horowitz, The Role of dipole charges in black hole thermodynamics, Phys. Rev. D 73 (2006) 024015 [hep-th/0505278] [INSPIRE].

[38] G. Compere, Note on the First Law with p-form potentials, Phys. Rev. D 75 (2007) 124020 [hep-th/0703004] [INSPIRE]. 
[39] Y. Tachikawa, Black hole entropy in the presence of Chern-Simons terms, Class. Quant. Grav. 24 (2007) 737 [hep-th/0611141] [INSPIRE].

[40] A. Fontanella and T. Ortín, On the supersymmetric solutions of the Heterotic Superstring effective action, JHEP 06 (2020) 106 [arXiv:1910.08496] [INSPIRE].

[41] E. Bergshoeff and M. de Roo, Supersymmetric Chern-Simons Terms in Ten-dimensions, Phys. Lett. B 218 (1989) 210 [INSPIRE].

[42] R.M. Wald, General Relativity, The University of Chigago Press (1992) [DOI] [InSPIRE].

[43] I. Racz and R.M. Wald, Global extensions of space-times describing asymptotic final states of black holes, Class. Quant. Grav. 13 (1996) 539 [gr-qc/9507055] [InSPIRE].

[44] R.R. Khuri and T. Ortín, A Nonsupersymmetric dyonic extreme Reissner-Nordstrom black hole, Phys. Lett. B 373 (1996) 56 [hep-th/9512178] [INSPIRE].

[45] J.D. Edelstein, K. Sfetsos, J.A. Sierra-Garcia and A. Vilar López, T-duality equivalences beyond string theory, JHEP 05 (2019) 082 [arXiv: 1903.05554] [INSPIRE].

[46] T. Azeyanagi, R. Loganayagam, G.S. Ng and M.J. Rodriguez, Covariant Noether Charge for Higher Dimensional Chern-Simons Terms, JHEP 05 (2015) 041 [arXiv:1407.6364] [INSPIRE].

[47] P.A. Cano, T. Ortín, P.F. Ramírez, A. Ruipérez and M. Zatti, $\alpha^{\prime}$-corrected, extremal, 5- and 4-dimensional black holes, work in progress. 